\title{
Gradhiva
}

GRADHIV

Revue d'anthropologie et d'histoire des arts

$14 \mid 2011$

Carl Einstein et les primitivismes

\section{À la recherche de l'Afrique dans Negerplastik de Carl Einstein}

Looking for Africa in Carl Einstein's Negerplastik

\section{Z.S. Strother}

Traducteur : Camille Joseph

\section{OpenEdition}

\section{Journals}

Édition électronique

URL : http://journals.openedition.org/gradhiva/2130

DOI : 10.4000/gradhiva.2130

ISSN : $1760-849 x$

Éditeur

Musée du quai Branly Jacques Chirac

Édition imprimée

Date de publication : 30 novembre 2011

Pagination : 30-55

ISBN : 978-2-35744-046-3

ISSN : 0764-8928

\section{Référence électronique}

Z.S. Strother, «À la recherche de l'Afrique dans Negerplastik de Carl Einstein », Gradhiva [En ligne], 14 | 2011, mis en ligne le 30 novembre 2014, consulté le 01 mai 2019. URL : http:// journals.openedition.org/gradhiva/2130 ; DOI : 10.4000/gradhiva.2130 


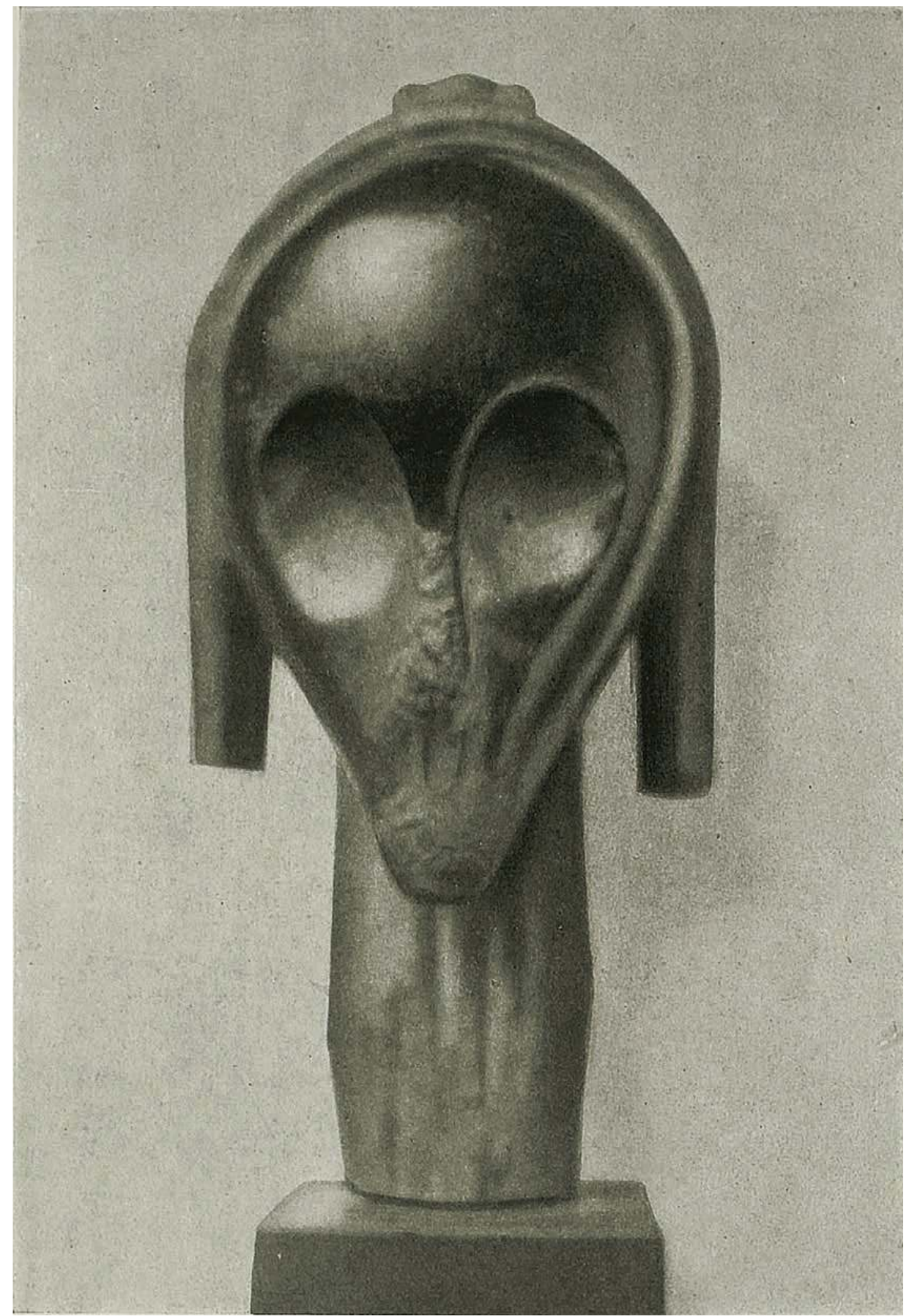

Fig. 1 Carl Einstein, Negerplastik, planche 18. Tête de reliquaire fang, Gabon, photo Alberto Ricci. 


\section{À la recherche de l'Afrique dans Negerplastik de

\section{Z.S. Strother}

* Se reporter aux p. 257-259 pour la bibliographie de cet article.

$\bullet \bullet$

1. Voir les comptes rendus de Negerplastik (1915-1920) reproduits dans Baacke 1990 , p. 85-133. Les éditions Kurt Wolff ont réédité une seconde version plus modeste de Negerplastik en 1920.

2. Jaquette de la traduction française de Liliane Meffre.

3. Patricia Leighten, qui a étudié les articles sur l'Afrique dans la presse française autour de 1905-190?, est une des rares exceptions (Leighten 1990).

4. Joyce Cheng émet l'hypothèse que les « réflexions [d'Einstein] sur l'usage social des objets, en particulier leur fonction rituelle », sont centrales dans son projet théorique d'«immanence métaphysique » (2009 : 87-97). Comme on le verra plus clairement dans la suite, c'est sur l'attribution des sources d'Einstein que nos points de vue divergent, ce qui nous amène à tirer des conclusions très différentes sur le rôle de l'Afrique dans son travail.
En 1914, deux hommes avaient le projet de publier, chacun de leur côté, le premier traité théorique sur l'art africain écrit par un Européen. Le peintre letton Voldemārs Matvejs et l'écrivain allemand Carl Einstein travaillèrent pour ainsi dire simultanément, sans se connaître. Matvejs mourut brutalement en mai et la publication de son manuscrit, Iskusstvo Negrov (L'Art nègre), fut retardée jusqu'en 1919. La Lettonie faisait alors partie de l'Empire russe et le peintre écrivait en russe sous le pseudonyme de "Vladimir Markov». Son texte, publié aux lendemains de la Révolution, exerça une forte influence sur l'avant-garde soviétique, sur Kazimir Malevitch, Vladimir Tatlin et Aleksandr Rodchenko, par exemple, avant d'être mis au rebut par les institutions artistiques soviétiques dans les années 1930. Le livre d'Einstein, Negerplastik (La Sculpture nègre), parut en 1915 et rencontra un vif succès, mais il tomba lui aussi peu à peu dans l'oubli'. Depuis 1961, ce texte a suscité un regain d'intérêt car son œuvre théorique éveille une curiosité grandissante. Coiffés de l'étiquette de "découvreur de l'art africain ${ }^{2}$ ", ces deux hommes sont regardés comme les héros virils d'un monde multiculturel prêt à les canoniser au xxie siècle.

Mais quel rôle joua l'Afrique dans leurs théories sur l'art africain? Simon Gikandi émet cette prévention : "On a beaucoup écrit sur Picasso et le primitivisme, mais peu sur sa relation spécifique avec l'Afrique3.» (2006 [2003] : 33) Il laisse entendre par là que les chercheurs ont repris à leur compte le soin qu'avait mis Pablo Picasso à séparer les œuvres d'art des peuples et des sociétés qui les avaient produites et «à minimiser le rôle constitutif de l'Afrique dans la fabrication du modernisme » (ibid. : 34). Les interrogations sur la place de l'Afrique chez Picasso peuvent être élargies à la communauté des modernistes européens fascinés par les objets d'art en provenance d'autres régions du monde. Le présent article reprend la question de Gikandi et s'interroge sur le rapport d'Einstein à l'Afrique, ainsi que sur l'origine de ses conceptions ${ }^{4}$. 


\section{CARL EINSTEIN ET LES PRIMITIVISMES}

La première vie de Negerplastik : les photographies

"Un nouvel accroc dans le canon de la beauté classique»

Hermann Hesse

Matvejs et Einstein constatèrent d'emblée qu'ils ne pouvaient analyser l'art africain sans commencer par réunir un corpus substantiel d'images. Comme Matvejs l'a souligné au début de son livre, il existait très peu de photographies de sculptures africaines isolées à l'époque. Par suite, il n'eut pas d'autre choix que d'effectuer plusieurs voyages à travers toute l'Europe et de photographier lui-même des œuvres remarquables conservées dans les collections des musées européens (Markov 2009 [1919] : 79-80). Einstein, quant à lui, profita de ses connexions avec le monde de l'art pour rassembler des photographies prises par des professionnels. Les deux livres dont il est ici question confirment la thèse de Frederick Bohrer selon laquelle la photographie joua un rôle essentiel dans l'invention de l'histoire de l'art parce qu'elle fut capable de générer des comparaisons et, comme l'affirme Bernard Berenson, d'autoriser une meilleure "expérience de l'art» (2002 : 248249) en donnant accès à des œuvres que les spectateurs n'auraient normalement pas pu voir ou en tout cas bien voir.

Negerplastik fut publié avec 119 photographies en noir et blanc représentant 94 sculptures différentes $5.80 \%$ des objets n'étaient figurés qu'avec une seule vue, de face ou de trois quarts. Les œuvres étaient pour la plupart prises sous un angle permettant de les photographier de plain-pied. Sous un éclairage soigné, la sculpture se présentait le plus souvent comme une série de plans entrecroisés (fig. 2). Ces images dégageaient une émotion cérébrale et un peu froide. Einstein travailla principalement sur des collections privées et, à quelques exceptions près, les objets avaient été dépouillés et ne laissaient plus voir que le travail du bois. Les lames du nkisi nkondi avaient ainsi été retirées (fig. 3 et 4), de même que les tissus, les chapeaux et collerettes de raphia de divers objets, et les couleurs vives avaient été estompées (fig. 5 et 6). Cette présentation systématique transforma un ensemble d'objets modifiés en provenance d'une vingtaine de pays en un corpus nommé «art africain » qui n'existait littéralement pas auparavant.

Il est intéressant de comparer le mode de présentation des objets de Negerplastik avec celui de livres antérieurs. Dans les Notes analytiques sur les collections ethnographiques publiées par le musée du Congo en 1906, près de 700 photographies étaient reproduites sur un papier à fort grammage (fig. 7). Ce fut l'une des premières et des plus luxueuses publications dédiées à la culture visuelle de l'Afrique. L'éclairage direct était également réparti sur toute la surface de l'objet et chaque sculpture était présentée, comme il se doit, avec des vues comparatives, deux vues de face et de profil, dans des reproductions en très haute résolution. En outre, les dimensions exactes auxquelles les sculptures avaient été réduites sur les photographies avaient été soigneusement calibrées, permettant ainsi d'évaluer ressemblances et différences sur une base scientifique.

La mise en page de Negerplastik reposait également sur un principe comparatif, mais la stratégie était radicalement différente. Les images placées les unes à côté des autres invitaient à établir des comparaisons formelles entre deux ou trois objets; cette opération était facilitée par le recours à une échelle, un éclairage et une perspective uniformes, qui faisaient ressortir les similarités et les différences. Le spectateur était par exemple incité à comparer deux masques 
Fig. 2 Carl Einstein, Negerplastik,

planche 71.

Statuette sénoufo, photo Alberto Ricci.

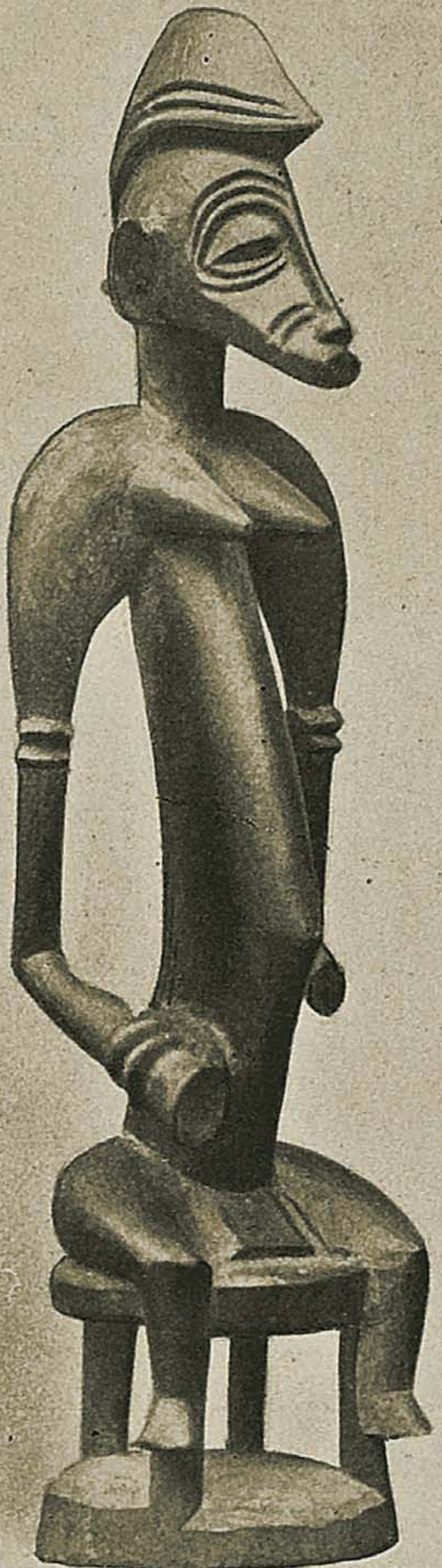




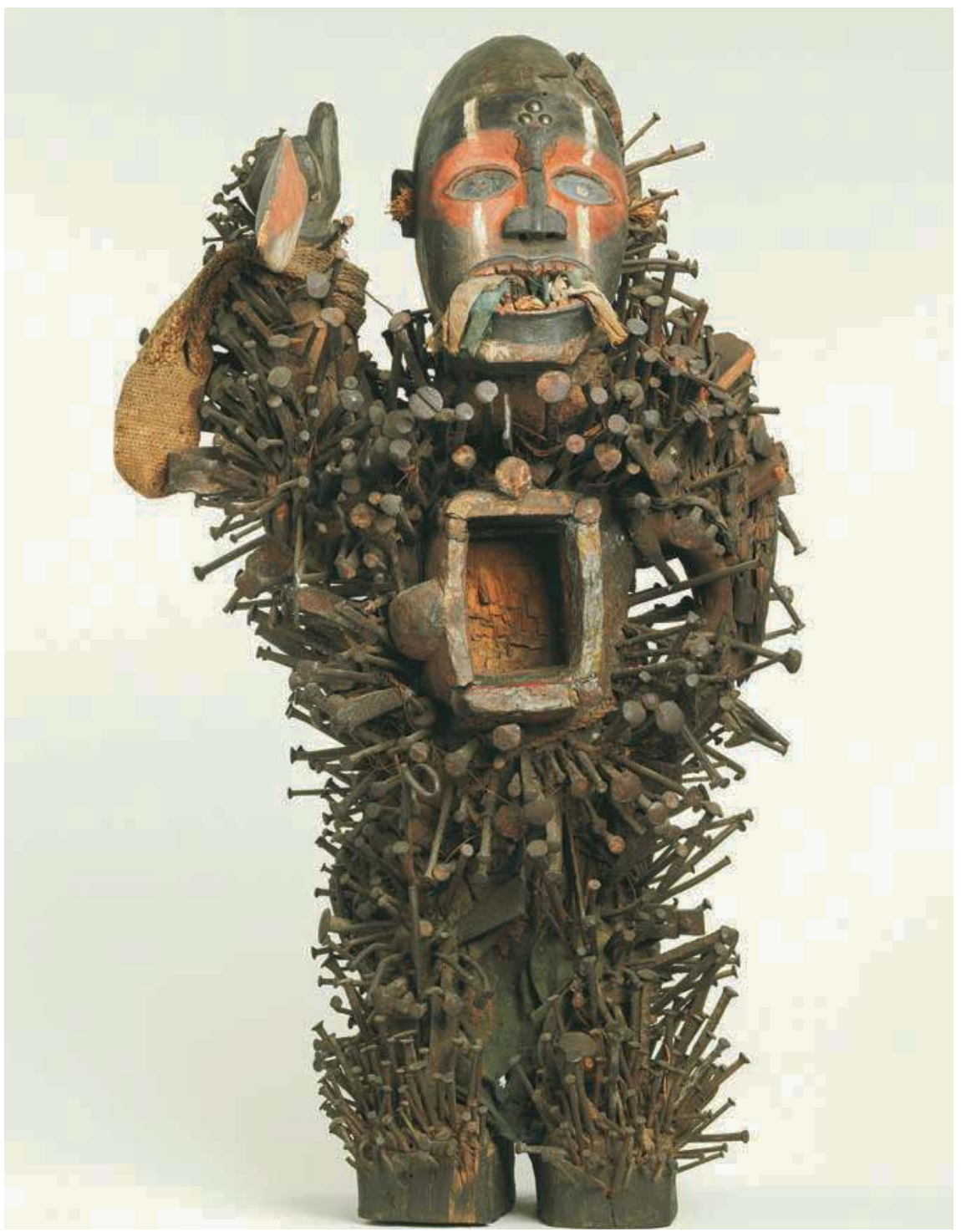

Fig. 3 Nkisi nkondi, Kongo, avant 1892 @ musée du quai Branly, photo Thierry Ollivier, Michel Urtado.

représentés à la même échelle (en apparence) et présentant la même patine (fig. 8). Dans les deux cas, les traits du visage étaient répartis le long de deux lignes formant une croix, qui divisait la figure en quatre carrés. La croix correspondait à une scarification prolongeant la ligne verticale du nez et la ligne horizontale des paupières. Cette logique structurelle commune révélait la différence entre les deux masques par un jeu de contraires : yeux convexes, yeux concaves; bouche ouverte, bouche fermée; présence d'oreilles, absence d'oreilles; etc. La manière dont les objets étaient présentés construisait artificiellement un rapport entre deux œuvres d'art distinctes.

Il faut noter que 17 sculptures étaient présentées sous plusieurs angles 6 . Mais

6. Treize objets sont présentés avec deux vues, trois objets avec trois vues, et un objet de Madagascar avec cinq vues. les strictes vues de profil étaient rares et n'étaient utilisées que pour produire un effet de surprise dans la composition visuelle. Negerplastik se détournait ainsi des modèles scientifiques et invitait au contraire à la «rêverie " poétique (Gros- 


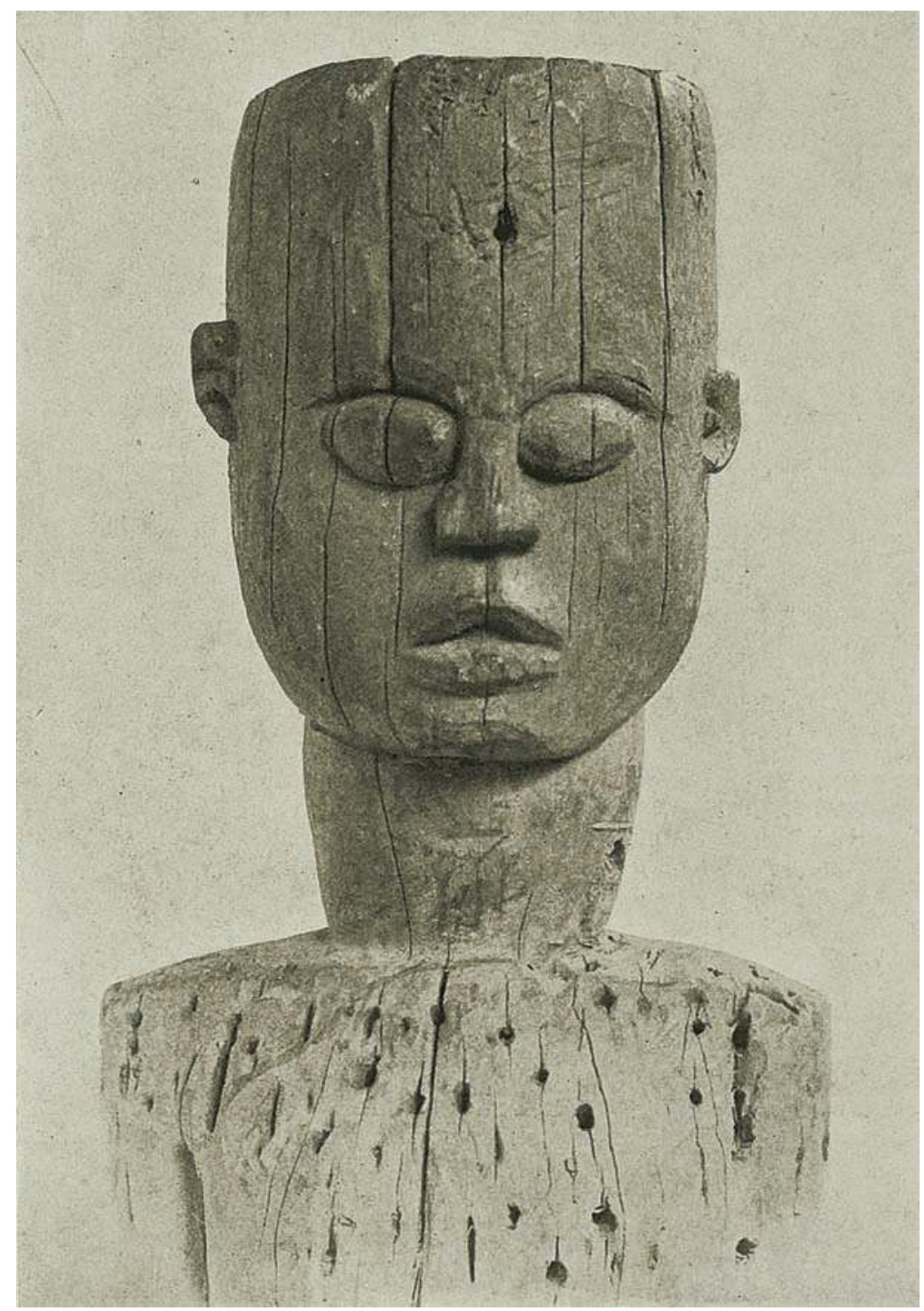

Fig. 4 Carl Einstein, Negerplastik, planche 19. Nkisi nkondi, Kongo, photo Alberto Ricci.

sman, 2007: 296) à travers toute une variété de techniques : en privilégiant une mise au point floue, en faisant flotter dans l'espace les objets dont l'ombre n'était que suggérée, en les éclairant au spot pour renforcer autant que possible l'aspect luisant de la patine.

André Malraux a brillamment montré que la circulation des photographies d'objets avait induit, de façon significative, un processus d'« intellectualisation de l'art ». Mary Bergstein résume ainsi son propos : la photographie de sculptures fut à l'origine d'"un ensemble homogène d'images " qui permit de comparer et de mettre en contraste les œuvres d'art entre elles «d'une manière presque mathématique " (1992: 476). Les images ont élevé le degré d'intimité entre spectateur et œuvres en donnant un accès uniforme à l'objet, sans considération de l'échelle ou de l'environnement initial (ibid.). Elles ont soustrait les œuvres à leur contexte géographique d'origine (ibid.). Dans le cas de Negerplastik, il 


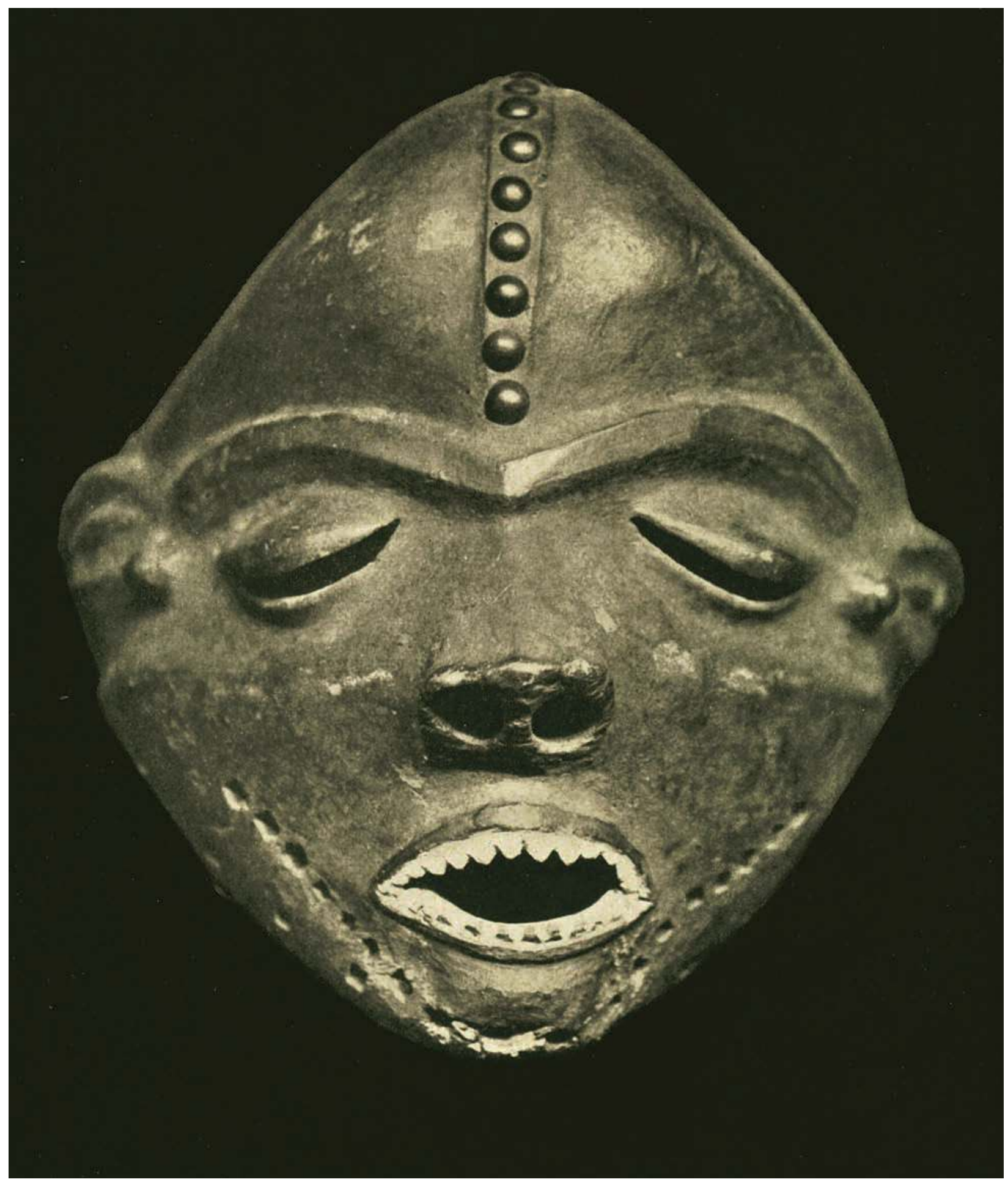

Fig. 5 Carl Einstein, Negerplastik, planche 92. Masque pende, photo Alberto Ricci. 


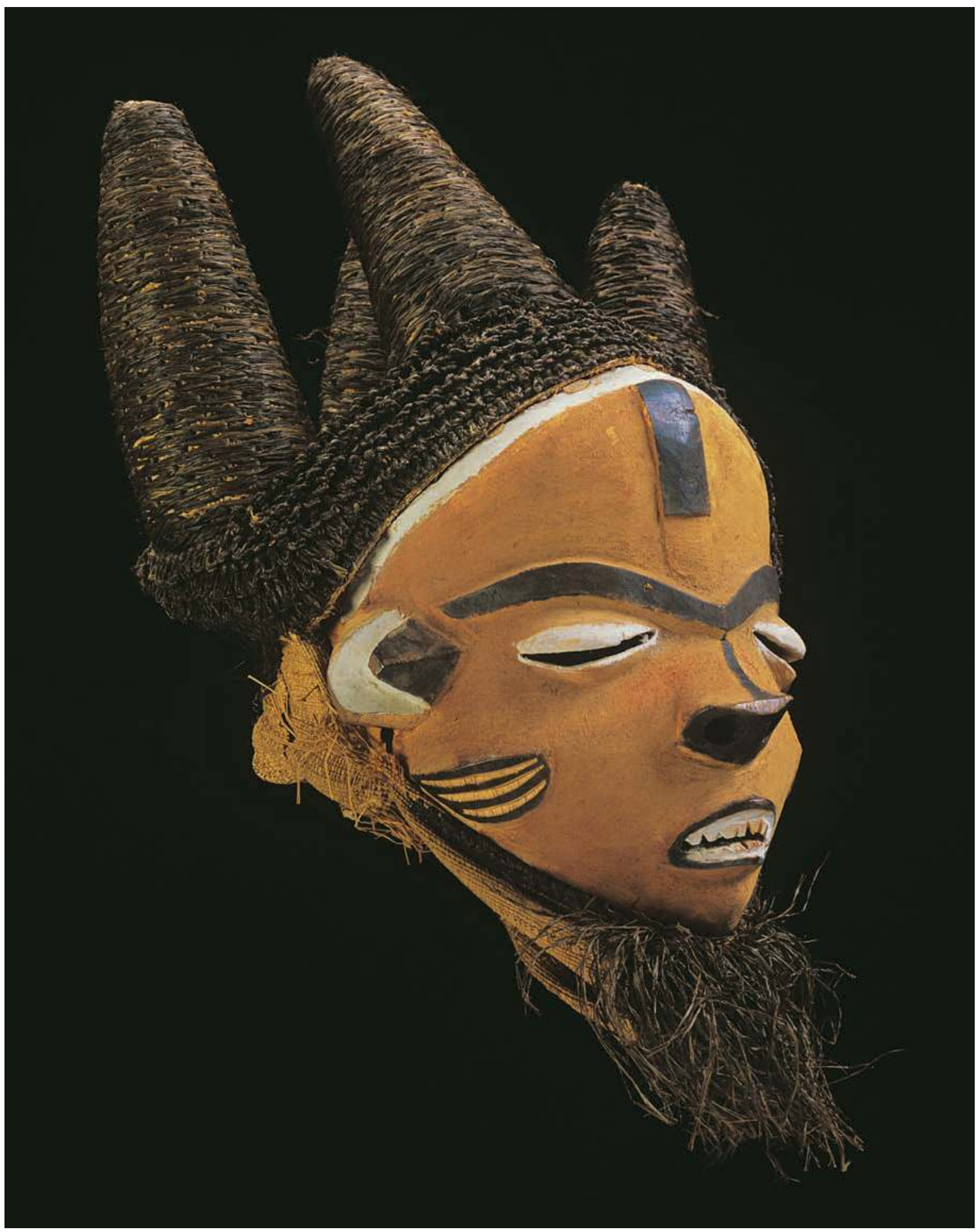

Fig. 6 Fumu (le chef), Pende Central. Artiste : Gabama a Gingungu, collection Musée royal de l'Afrique Centrale, Tervuren, Belgique (c) MRAC Tervuren, photo J.-M Vandyck. 


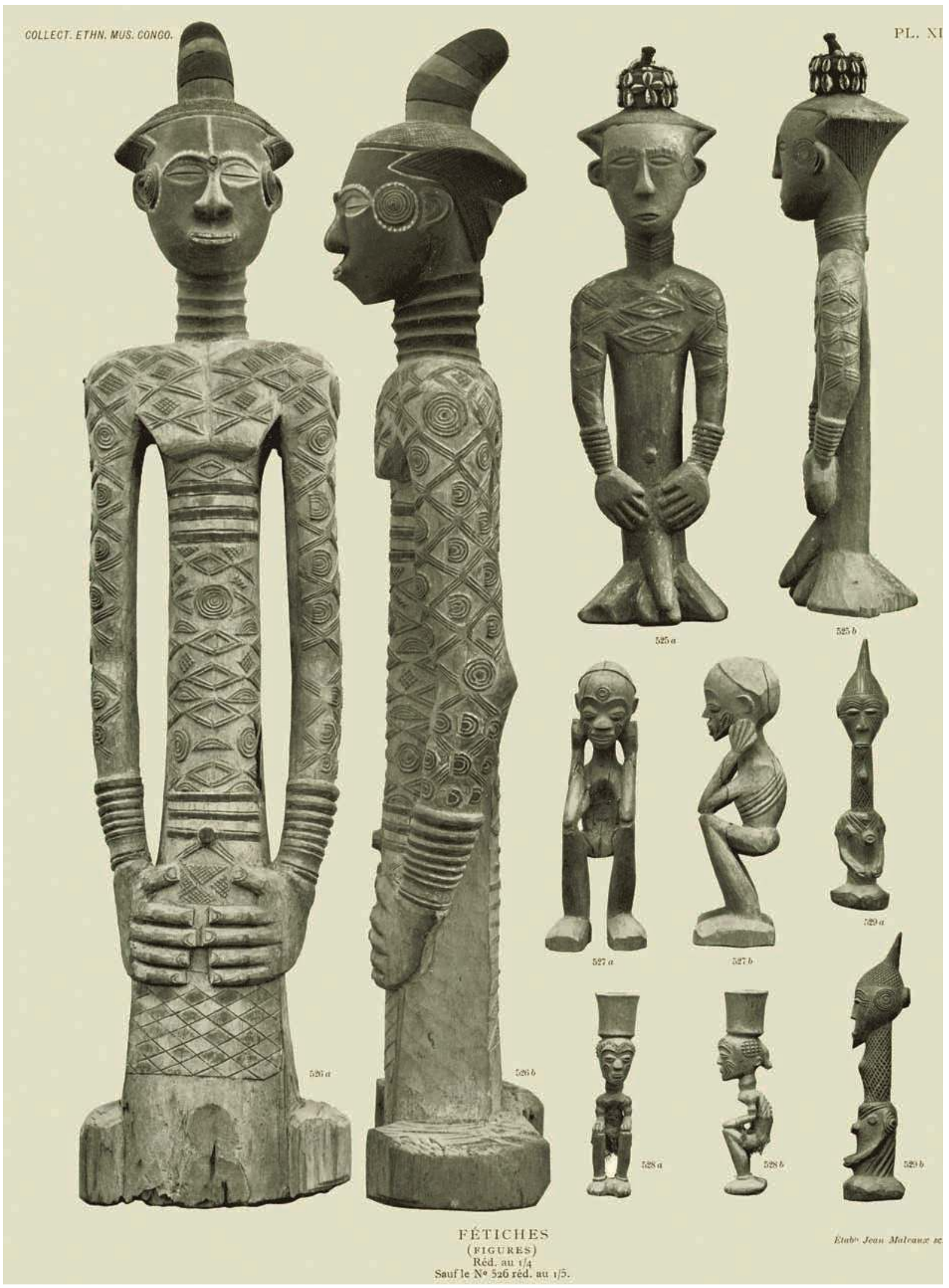

Fig. 7 Notes analytiques sur les collections ethnographiques. Annales du musée du Congo (Tervuren), in Ethnographie et Anthropologie (série 3), tome I, fasc. 2 (Les Arts - Religion), juillet 1906, planche 40. 
importait peu que l'œuvre provienne du Gabon ou du Congo belge, qu'elle soit issue de la forêt ou de la savane, qu'elle ait été destinée à être montrée en public ou réservée à un usage domestique : toutes ces distinctions étaient effacées au profit de la recherche "des formes plastiques pures» (Einstein, 1915: xI; trad. Meffre, 1998 : 27).

L'impression d'unité stylistique qui ressort de la mise en page de Negerplastik est si forte qu'il est bon de se rappeler la mise en garde d'Allan Sekula : les " projets d'archivage " peuvent donner l'illusion trompeuse d'une véritable cohérence à cause du grand nombre d'images rassemblées. La vérité photographique ne surgit pas ici du raisonnement mais de l'expérience (1983: 199). C'est l'archive qui «dégage» du sens à partir de l'usage, qui extrait l'objet de son contexte afin d'«établir une relation d'équivalence visuelle abstraite entre les images " (ibid. : 194-195). Autour de 1915-1920, un petit nombre de critiques surent résister à la logique qui sous-tendait ce recueil de «sculpture nègre». Sascha Schwabacher tourna en dérision le flou du concept, le comparant à une catégorie aussi générale que la "sculpture indo-européenne ». Elle s'interrogea sur la pertinence de la réunion dans un même ensemble d'œuvres du Bénin, du Congo, de Mélanésie et de Polynésie (in Baacke, 1990 : 120). Pour Viktor Christian, le projet aurait gagné en précision s'il avait été limité géographiquement aux Kulturkreisen (aires culturelles) d'Afrique de l'Ouest (in Baacke, $1990: 128$ ). Cependant, la plupart des spécialistes de l'époque acceptèrent sans discuter la cohésion de l'ensemble et Einstein fut victime de son propre succès. Plus tard, il écrivit qu'« un fait » compensait le "pénible sentiment d'incertitude » entourant l'art africain : l'« unité de style» (1922 [1921] : 6).

Hermann Hesse a décrit l'effet que produisirent sur lui les archives photographiques de Negerplastik, qui décentrèrent ses attentes vis-à-vis de l'art. «En vérité, écrivait-il, je ne peux pas dire que je trouve belles les sculptures nègres. » Néanmoins, Einstein l'avait convaincu que, si l'on pouvait trouver « cet art étranger et perturbant", personne n'était en droit de lui refuser le statut d'« art [...] légitime et pleinement justifié en lui-même. Un nouvel accroc au canon de la beauté classique" (in Baacke, 1990 : 96). Selon l'artiste et critique tanzanien Everlyn Nicodemus, on peut mettre ce résultat au crédit d'Einstein; il reconnaît dans le même temps n'avoir pris conscience qu'en Europe de l'existence de ce qu'on appela plus tard «l'art africain classique»: «Je suis allé voir les sculptures dans les musées de Paris et de Londres. Même aujourd'hui, je ne peux expliquer ce que j'ai ressenti devant ces œuvres : elles représentaient une Afrique inconnue; peut-être ne m'avaient-elles pas parlé parce que je viens d'une région qui ne connaît pas de tradition de sculpture ${ }^{8}$." (Nicodemus, 1993:32) Les archives photographiques de Negerplastik ont défini le canon de l'art africain à partir de sculptures de bois provenant dans leur grande majorité des colonies françaises et belges.

Qui se trouvait derrière les 119 photographies, publiées sans aucune légende et sans correspondance directe avec le texte ${ }^{9}$ ? Nous ne le saurons peut-être jamais. Einstein fut engagé volontaire pendant la Première Guerre mondiale et fut gravement blessé à la tête en novembre 1914 (Meffre, 2002 : 52, 53). Dans une lettre écrite en français, il faisait part de ses regrets : «Mon premier bouquin c'est un torse [fragment] parce que c'était publié par l'éditeur pendant que j'étai[s] au lazareth [sic]. " Dans cette même lettre, il demandait à son correspondant de lui faire parvenir des photographies d'objets de sa collection personnelle en lui promettant de les publier dans son prochain livre (in Baacke, 1980 : 142; Bassani, 1998 : 102). Il revenait en effet bien trop cher d'engager un photographe professionnel.

\footnotetext{
-

7. Christian faisait ici référence au modèle de Leo Frobenius des «aires culturelles », à savoir des régions ayant en commun un ensemble de traits culturels visibles dans le style ou bien définis historiquement (Frobenius, 1898).

8. Je remercie Sebastian Zeidler d'avoir attiré mon attention sur ce texte.

9. Plusieurs comptes rendus déplorèrent l'omission de la date, de la provenance et de l'origine ethnique des objets (voir Baacke, 1990 : 104, 108, 128]. Einstein apporta un soin particulier aux légendes accompagnant les illustrations de son second ouvrage, Afrikanische Plastik.
} 


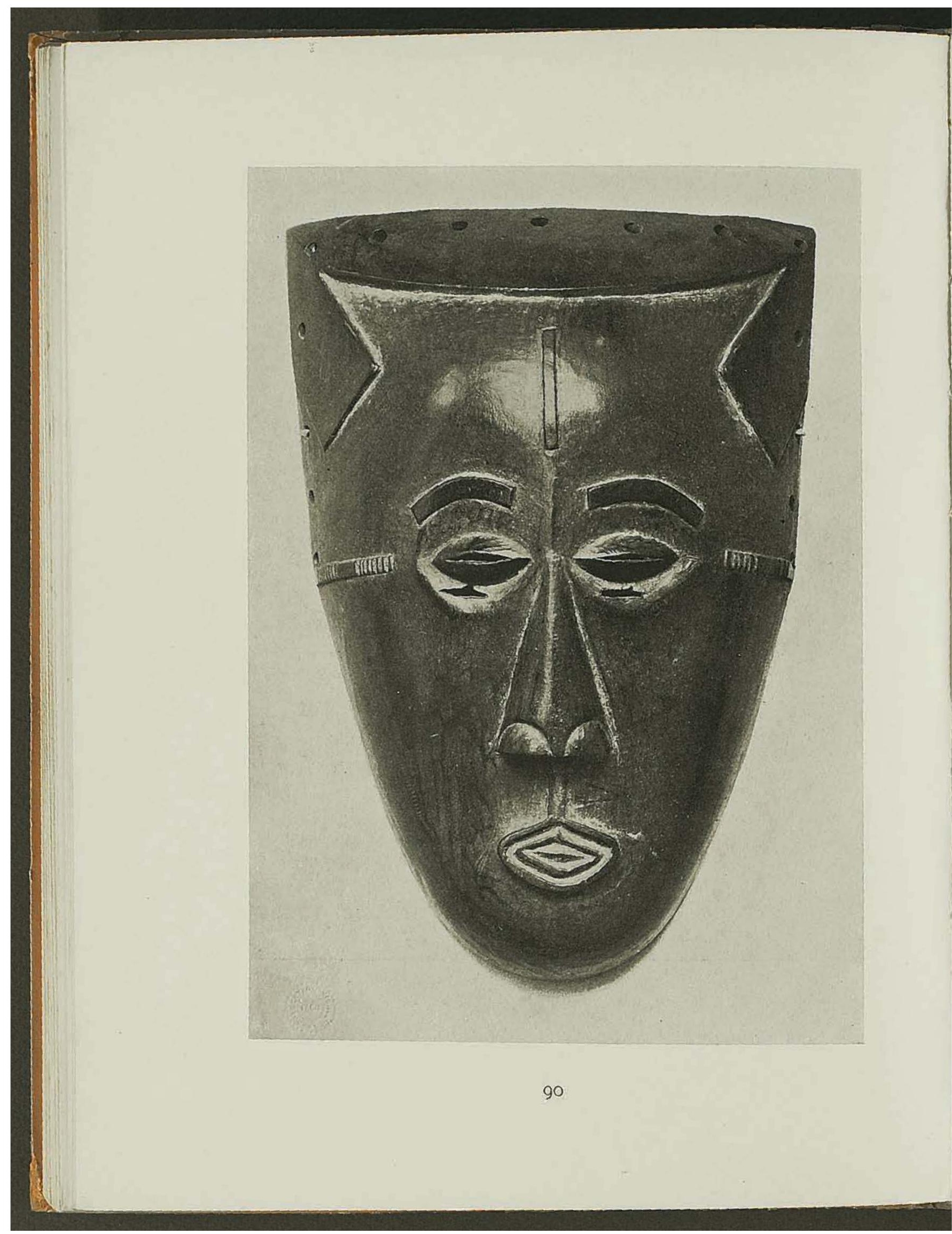

Fig. 8 Carl Einstein, Negerplastik, planches 9o-91. À gauche : masque kuba ou lele, avec un tampon "Collection [Charles] Vignier". À droite : masque d'origine inconnue, nord-est du Congo? 


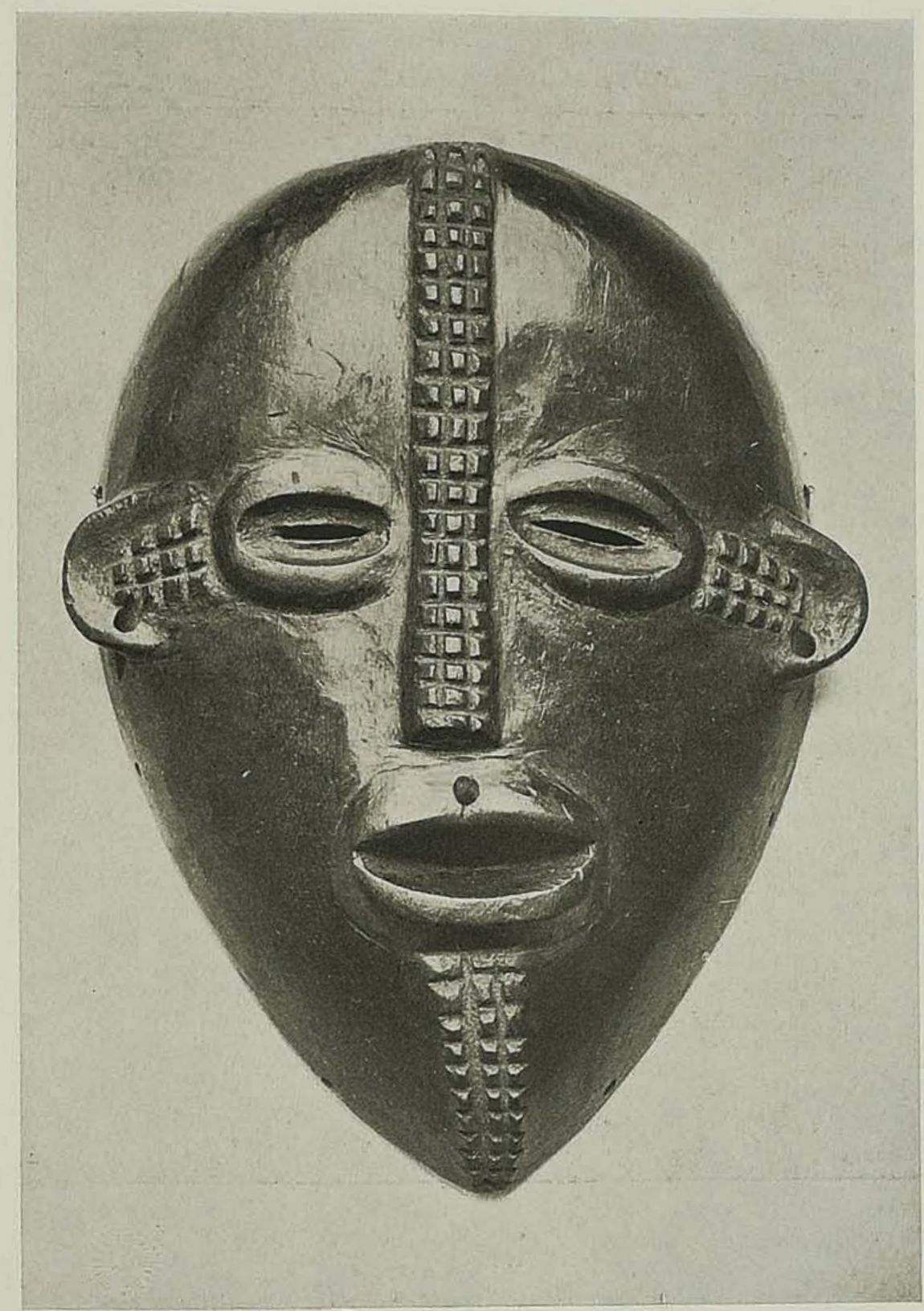




\section{CARL EINSTEIN ET LES PRIMITIVISMES}

- -

10. Ce fut notamment le cas de Frank Haviland, qui fit photographier sa collection par Druet avant 1914. Certaines de ces photographies ont été reprises dans Negerplastik (Laude, 1968 : 115 ; Bassani, 1998 : 106, 110).

11. Paudrat, 1984 : 144, 151 ; Bassani, 1998. Selon Paudrat et Bassani, quatorze objets proviennent directement de la collection de Brummer. Pour le reste, une grande partie appartenait aux clients les plus importants du marchand d'art. En 1913, Brummer avait mis à la disposition de la revue d'avantgarde tchèque Umelecky mesicnik neuf photographies représentant six objets de sa collection, qui furent ensuite reprises dans Negerplastik (planches 6, 16, 17, 46, 57, 86, 87, 99, 100; Bassani, 1998 : 114-120).

12. Un des textes promotionnels de 1915, par exemple, annonçait «119 planches à grande échelle et d'une excellente qualité [...], présentées dans une mise en page pédagogique » (in Baacke, 1990 : 112]. Bassani fait cependant remarquer qu'il ne discerne aucune logique dans la mise en page en termes d'ethnie, de région, de fonction ou de style - alors qu'il en va tout autrement dans le volume plus tardif d'Einstein, Afrikanische Plastik (Bassani, 1998 : 102). L'agencement des images selon une logique différente dans le second livre explique peut-être pourquoi les juxtapositions sont d'une banalité singulière.

13. L'expression est de Friedrich Markus Huebner (in Baacke, 1990 : 110).
En 1913, les plus grands collectionneurs et marchands d'art africain faisaient photographier leurs objets pour des motifs promotionnels et pour échanger des informations ${ }^{10}$. Les images rassemblées par Einstein proviennent sans doute de diverses sources, mais leur similitude suggère qu'il existait alors dans cette communauté un style codifié de présentation de l'« art primitif ». Cette difficulté d'obtention des photographies semble donner raison à Hans Purrmann, selon lequel le marchand d'art Josef Brummer fut l'« instigateur » de Negerplastik (in Baacke, 1990 : 87). Selon Jean-Louis Paudrat, Brummer aurait non seulement fourni la grande majorité des images du livre, mais également financé sa publication ${ }^{11}$.

À l'époque de la parution de Negerplastik, le graphisme allemand était de très haute qualité et Wendy Grossman a justement attiré l'attention sur «les juxtapositions ingénieuses» induites par «la mise en page du livre» (2007: 296). La première édition mesurait $25 \times 9 \mathrm{~cm}$ et a été imprimée sur papier couché à grammage élevé. C'est certainement le graphiste qui retoucha les photographies afin de renforcer leur uniformité et qui fut responsable de la logique formelle de l'ensemble (fig. 9). Les textes promotionnels de l'éditeur mettaient l'accent sur les reproductions, leur nombre, leur taille et leur qualité1 ${ }^{12}$. La qualité supérieure de l'impression et de la mise en page faisait toute la différence. Malgré les photographies très soignées de Matvejs, Iskusstvo Negrov fut publié sur un papier de mauvaise qualité qui jaunissait rapidement, ce qui réduisit considérablement son impact potentiel.

À la sortie de Negerplastik, un nombre impressionnant de comptes rendus parurent dans des supports culturels très variés, témoignage de l'actualité de son objet. De nombreux lecteurs firent une critique approfondie, voire acerbe, de la méthode de l'auteur, mais tous étaient d'accord pour reconnaître au livre une valeur en tant qu'“atlas d'images ${ }^{13}$ " (Bilderatlas). De fait, plusieurs chercheurs ont noté que les planches sur papier glacé de Negerplastik eurent à l'origine un impact plus grand que le texte lui-même et que beaucoup d'artistes européens qui ne lisaient pas l'allemand les avaient utilisées (Zeidler, 2004 : 122; Grossman, 2007: 297-299, 328). Du point de vue qui m'occupe ici, la création d'archives d'images homogènes, agencées avec soin pour permettre la comparaison et le contraste, constitue avant tout l'acte fondateur de l'histoire de l'art africain.

\section{Avant et après Bruxelles \\ «[I]ci, je négrifie complètement. [E]xcès africain. »}

Que signifiait écrire un livre sur l'art africain autour des années 1913-1914? Nombreux sont les chercheurs qui ont fatalement voulu projeter sur les premiers écrits d'Einstein ce que l'on sait de l'homme qui travailla avec Michel Leiris dans Documents; celui qui combattit pour la cause républicaine en Espagne; et celui qui fut traqué à mort par les nazis en France en 1940. Pour connaître la genèse et mesurer l'originalité et l'excentricité complète de Negerplastik, il faut suivre de près la trajectoire du développement intellectuel d'Einstein.

Einstein commença par se faire un nom d'écrivain en publiant Bebuquin oder die Dilettanten des Wunders (Bebuquin ou les dilettantes du miracle) en 1912. À cette époque, il vivait surtout de la publication de critiques couvrant tout un pan de la vie artistique de son temps. À partir de 1913, il commença à se concentrer davantage sur la critique d'art, au moment où parurent les premières références connues sur l'art africain. En août 1913, il écrivit au directeur du département 

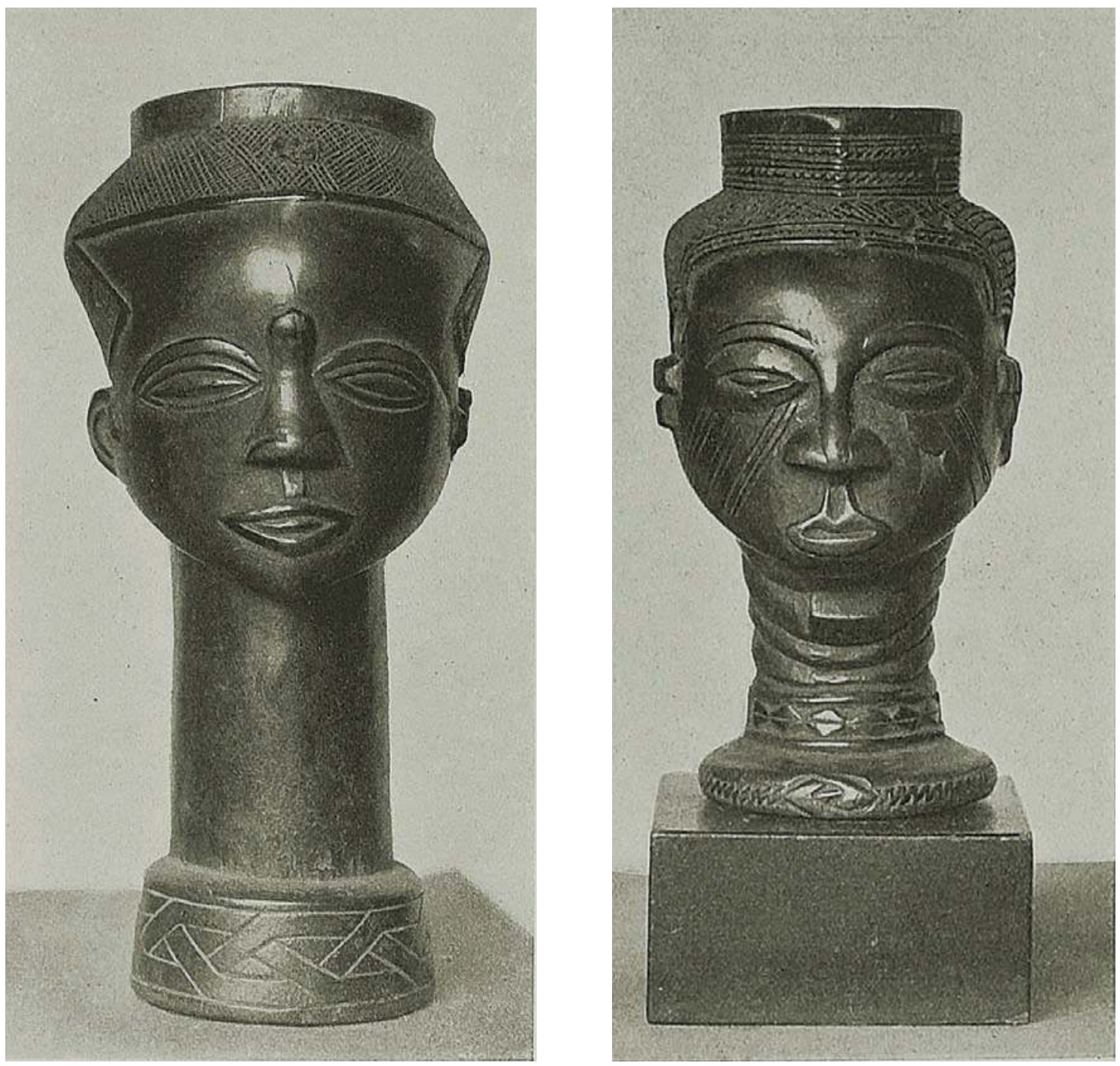

Fig. 9 Carl Einstein, Negerplastik, planches 74-75, Coupes à boire kuba. Berlin, Museum für Völkerkunde \#III C 19637.

d'Anthropologie du Museum für Völkerkunde de Berlin et sollicita son aide. Il évoqua l'enthousiasme des plus grands artistes européens pour l'«art primitif » et proposa d'y consacrer un supplément spécial de la revue Der Merker, dans lequel seraient reproduites «quelques-unes des pièces merveilleuses » du musée. Le but officiel était d'attirer l'attention des collectionneurs allemands d'art moderne sur «l'immense valeur artistique des sculptures nègres [et] des œuvres mexicaines " (in Baacke, $1990: 136$ ).

Le supplément de Der Merker ne vit jamais le jour et, très vite, Einstein définit plus précisément ses intérêts. En novembre 1913, il participa à l'organisation d'une exposition à la Neue Galerie de Berlin qui présentait des œuvres de Picasso, André Derain et Henri Matisse et, dans une pièce voisine, des sculptures africaines (Neumeister, 2008 : 173-175, 178). En décembre 1913, il intégra ce qu'un commentateur désigna comme " une série de sculptures nègres superbes » dans une rétrospective de l'œuvre de Picasso de 1901 à 1912 (in ibid. : 175, 182 n. 12, 13). 
-

14. Einstein emmena même les enfants d'un de ses amis écrivain en visite à la bibliothèque en avril 1916, après avoir découvert qu'ils partageaient sa passion pour les choses de l'Afrique (Meffre, 2002: 62).
Selon Heike Neumeister, le rôle que joua Einstein par deux fois dans l'organisation de ces expositions fournit des informations cruciales sur l'état d'esprit qui était le sien juste avant et pendant l'écriture de Negerplastik (ibid. : 175). Il entretenait alors des relations avec des galeristes et des collectionneurs intrigués par l'enthousiasme des artistes d'avant-garde pour ce qu'on appelait «l'art primitif " et, en particulier, la sculpture africaine. Brummer était à Berlin au moment où eurent lieu les deux expositions mentionnées ci-dessus (ibid. : 182 n. 8) et leur collaboration supposée sur Negerplastik pourrait bien remonter à cette date. Au vu de cette chronologie, on peut penser qu'Einstein rédigea la plus grande partie de son texte dans la première moitié de 1914 .

En août 1914, il s'engagea comme volontaire dans le conflit mondial (s'opposant à son patron et beau-frère Franz Pfemfert, l'éditeur de Die Aktion; Meffre, 2002 : 52). Toute sa vie, Einstein fut attiré par le romantisme de la camaraderie virile des temps de guerre. Il écrivait à l'époque : "Nous entrions dans une nouvelle communauté humaine; des hommes qui voulaient mourir ou vaincre ensemble." (Ibid. : 52 n. 98) En novembre, il fut gravement blessé à la tête alors qu'il se trouvait en Belgique (ibid. : 58) et il passa quatre mois en convalescence dans un hôpital militaire de Berlin, de janvier à mai 1915. Comme on l'a dit plus haut, il regretta de s'être trouvé à l'hôpital au moment du montage de Negerplastik.

Après avoir repris du service en Alsace, Einstein fut transféré au printemps 1916 à la section coloniale de l'administration civile du gouvernement général de Bruxelles. Pour Liliane Meffre, la publication de Negerplastik ne fut sans doute pas pour rien dans sa nomination à ce poste, qui lui offrit des conditions de travail particulièrement avantageuses; cette période eut une importance décisive pour le travail ultérieur d'Einstein quand celui-ci aborda des thématiques africaines (ibid. : 62-66). Il appréciait beaucoup son officier supérieur, Edmund Brückner, administrateur de carrière dans le service colonial allemand et ancien gouverneur du Togo avant la guerre. Un homme ayant concrètement connu l'Afrique entrait enfin dans le cercle de ses connaissances (ibid. : 66).

En tant qu'officier colonial, Einstein pouvait facilement accéder à l'une des meilleures bibliothèques du monde sur l'Afrique, tout particulièrement sur l'art et la culture d'Afrique centrale, la bibliothèque du musée du Congo à Tervuren ${ }^{14}$. Dans son programme de relations publiques, le tristement célèbre État indépendant du Congo avait inclus la construction d'un important musée et le financement de plusieurs publications sur l'art et la culture, dont les somptueuses Notes analytiques évoquées plus haut. Quand le pays changea de mains en 1908, le Congo belge poursuivit son engagement en faveur de l'ethnographie et soutint la publication de quatre titres qui figurent dans la bibliographie du second livre d'Einstein sur l'art africain.

Einstein acquit la notoriété d'un auteur ayant publié sur l'Afrique et il lui arriva de tromper son monde en faisant croire qu'il s'était effectivement rendu sur le continent africain (ibid. : 62-65). Son succès aiguisa son appétit pour des projets plus ambitieux. De cette période a survécu une lettre au ton euphorique, dans laquelle Einstein écrivait à son patron Franz Blei, « du bureau du feu ministre belge des Colonies » : «je négrifie ici complètement. Excès africain [...]. Cette fois je vais rassembler l'Afrique dans deux livres, on trouvera même l'occasion de constater dans mon travail le sérieux typique de mon pays " (in Baacke, 1990 : 138-39; trad. Meffre, $2002: 65-66$ ). 
Le choix des termes est fascinant. Avec «je négrifie », Einstein laissait entendre qu'il passait le plus clair de son temps libre à lire et à penser à l'Afrique. Il promettait que, "cette fois-ci" (à la différence de Negerplastik), il irait "collecter » ou "rassembler" l'Afrique (Afrika...versammeln) avec le "sérieux typique de [son] pays ", c'est-à-dire l'Allemagne (die heimatliche Gründlichkeit).

Einstein fut en poste à Bruxelles du printemps 1916 au mois d'octobre 1917, et ses publications ultérieures montrent qu'il se rendait à la bibliothèque de Tervuren pour travailler. Son projet de "collecter" l'Afrique débuta par la recherche de légendes, auxquelles il s'intéressait depuis toujours. En 1916-1917, il se mit à publier des traductions en vers libres de chants, prières et mythes de différents peuples d'Afrique centrale ${ }^{15}$. En 1921, il confessa que sa quête de textes oraux était en partie fondée sur l'espoir qu'ils pourraient éclairer les arts visuels de ces mêmes cultures; force était cependant de constater qu'ils appartenaient en fait à «des courants traditionnels entièrement divergents » (1922 [1921] : 6). Curieusement, sa conclusion faisait écho à celle de Friedrich Markus Huebner qui, dans son compte rendu de Negerplastik de 1915, faisait un jeu de mots sur le titre du Und Afrika sprach de Leo Frobenius. Pour Huebner, il était tentant de chercher des relations entre " des idoles terrifiantes en bois peint aux couleurs criardes » et " des histoires de fantômes ou des formules magiques sculptées grossièrement ", mais la "sculpture nègre" et la "poésie nègre" appartenaient à des branches artistiques distinctes. Huebner concluait ainsi : " Leo Frobenius témoigne contre Carl Einstein ${ }^{16}$. ”

En octobre 1917, Einstein fut renvoyé sur le front, d'où il écrivit ces lignes à sa femme : «Je ne supporte plus la guerre. Tout s'effondre; tout ce qui m'importait est détruit. " (Meffre, 2002 : 73) Rapidement, Einstein fut à nouveau blessé et hospitalisé, sans doute pour traumatisme psychologique (ibid. : 73-75). Il semblerait que cette expérience ait entraîné un éveil de sa conscience politique, comme pour bien d'autres soldats (Kiefer, 1987: 149). Après la guerre, il soutint la ligue Spartakus, animée selon lui de «la volonté de rendre à l'être humain la possibilité d'une société humaine " (Meffre, 2002 : 88).

Que nous apprend cette chronologie? En 1913-1914, Einstein avait toujours des aspirations littéraires et évoluait dans un cercle de galeristes et de collectionneurs d'art moderne intéressés par l'«art nègre ». Il n'était pas encore véritablement actif sur le plan politique et il s'engagea volontairement dans l'armée allemande en dépit de l'opposition de sa famille. Rien n'indique qu'Einstein ait eu alors des lectures ou des réflexions très approfondies sur l'Afrique. L'affirmation selon laquelle Negerplastik «se place en dehors du discours colonialiste, il en est même une critique ", tient dès lors du vœu pieux (Kiefer, 1987 : 152).

Les choses changèrent en 1916-1917 avec le succès de Negerplastik et l'affectation d'Einstein à l'administration coloniale de Bruxelles, où il fut confronté à des hommes qui avaient une expérience directe du continent africain. Quand Einstein affirmait que, "cette fois", il allait travailler de manière approfondie à collecter l'«Afrique", il anticipait sur les différences radicales qui existent entre Negerplastik et sa suite, Afrikanische Plastik (1921). Le premier ouvrage se lit comme un travail de critique où Einstein apparaît sûr de lui; le second se présente comme un texte savant rigoureux et bien renseignéi. ${ }^{17}$ À la différence de Negerplastik, Afrikanische Plastik est truffé de références à des auteurs, de citations, de noms de lieux et de mots africains désignant différents genres de sculptures. La bibliographie fournie à la fin est soigneusement adaptée aux objets sélectionnés pour les illustrations et montre qu'Einstein maîtrisait les connaissances contemporaines sur le sujet ${ }^{18}$.
- -

15. Textes réédités dans Baacke, 1980 : 397-400, 414, 415, 421-437. En 1925, Einstein en publia une magnifique version augmentée, Afrikanische Legenden. Ses sources proviennent surtout de l'Afrique centrale francophone, à savoir des Luba, Holoholo, Kaniok, Kuba et Fang.

16. «Leo Frobenius zeugt wider Carl Einstein », in Baacke, 1990 : 110.

17. Klaus Kiefer a même noté un changement de style au profit de verbes de modalité comme scheinen ou mögen pour marquer les passages spéculatifs (1987: 156).

18. $60 \%$ des 23 titres cités furent publiés à partir de 1909. 


\section{CARL EINSTEIN ET LES PRIMITIVISMES}

- -

19. Einstein se montrait très prudent face au travail de Frobenius. Ce dernier avait une grande expérience de terrain, mais au vu de ses théories grandioses, de son style ampoulé, de ses publications populaires et de son manque de légitimité académique, sa réception au sein de l'université allemande ne pouvait être qu'ambivalente. Dans Afrikanische Plastik, la prédominance des Yoruba, le choix de certaines images et même la formulation de certaines légendes montraient qu'Einstein connaissait bien Und Afrika sprach.
Les chercheurs travaillant sur Einstein ont, sans exception, relevé la divergence entre les deux livres et ont disqualifié Afrikanische Plastik comme un texte "plus ethnographique"; mais ce jugement est trompeur. Einstein ne s'intéresse ni à la fonction des objets ni à la manière dont ils s'insèrent dans la praxis sociale. Il affirme clairement se donner pour mission «d'ouvrir la voie aux recherches spécialisées intéressant l'histoire de la sculpture et de la peinture", plutôt que d'alimenter des artistes européens en mal d'imagination (1922 [1921] : 3). Le projet révolutionnaire d'écrire une histoire de l'art pour l'Afrique avait peut-être été inspiré par des comptes rendus de Negerplastik, qui, dans bien des cas, appelaient de leurs vœux une méthodologie plus historique. Dans son second ouvrage sur l'art africain, Einstein tentait d'établir des relations historiques entre différentes traditions artistiques à travers une analyse visuelle d'objets spécifiques et s'efforçait dans la mesure du possible de fournir des dates précises. Il y privilégia l'art du portrait et souleva la question des rapports entre sculpture et peinture. Afrikanische Plastik n'était "ethnographique» que dans la mesure où Einstein faisait la part belle à des auteurs possédant une expérience de terrain significative plutôt qu'à des sources populaires comme les journaux, les guides de musées ou les récits de voyage.

\section{À la recherche de l'Afrique dans Negerplastik}

On ne trouve pas beaucoup trace de l'Afrique dans Negerplastik, avant Bruxelles. À cet égard, le texte d'Einstein représente un contraste significatif par rapport à son jumeau Iskusstvo Negrov, qui a la même visée théorique. Le texte de Matvejs s'ouvre sur une longue synthèse des publications de Frobenius, car l'auteur estimait que les artistes de langue russe devaient impérativement avoir accès à ce type de recherche de haut niveau. Les Européens avaient été sidérés par les découvertes archéologiques de l'ethnologue allemand au Nigeria en 1910-1912. De nos jours, la communauté scientifique éprouve, au mieux, à l'égard de Frobenius des sentiments mêlés mais, pour Matvejs, il était celui qui avait démontré que les Africains possédaient eux aussi une histoire (et une histoire de l'art) : «Il existe là un passé riche, puissant et fabuleux. " (Markov, 2009 [1919] : 84)

Il est difficile de croire que le Berlinois Einstein ne connaissait ni Und Afrika sprach, ensemble somptueux réunissant plusieurs volumes, ni certains des rapports qui commencèrent à voir le jour en $1912^{19}$. Negerplastik s'ouvre sur cette remarque plaintive et condescendante: "On pourra peut-être tirer des planches présentées dans cet ouvrage la conclusion suivante : le Nègre n'est pas un être non évolué; une culture africaine d'importance a disparu; le Nègre actuel correspond à un possible type "antique ", comme, peut-être, le fellah à l'Égyptien ancien. » (1915: v; trad. Meffre, 1998 : 18) D'un côté, la découverte à Ife de figures naturalistes de cuivre jaune et de terre cuite très achevées apportait la preuve que l'Afrique subsaharienne avait "dans le passé» été un lieu de civilisation. D'un autre côté, pour des observateurs bourgeois éduqués dans la tradition gréco-romaine, une telle découverte impliquait que cette civilisation avait disparu, en partie à cause du contact avec l'Europe : "Plus on pénètre profondément dans les strates des civilisations anciennes, plus on trouve des objets d'un raffinement extrême. Il s'ensuit qu'il existait durant l'Antiquité une culture ancienne, bien supérieure à celle que l'on trouve aujourd'hui sur le sol africain. » (Frobenius, 2009 [1912] : 195) Einstein n'accordait pas de primat au naturalisme, 


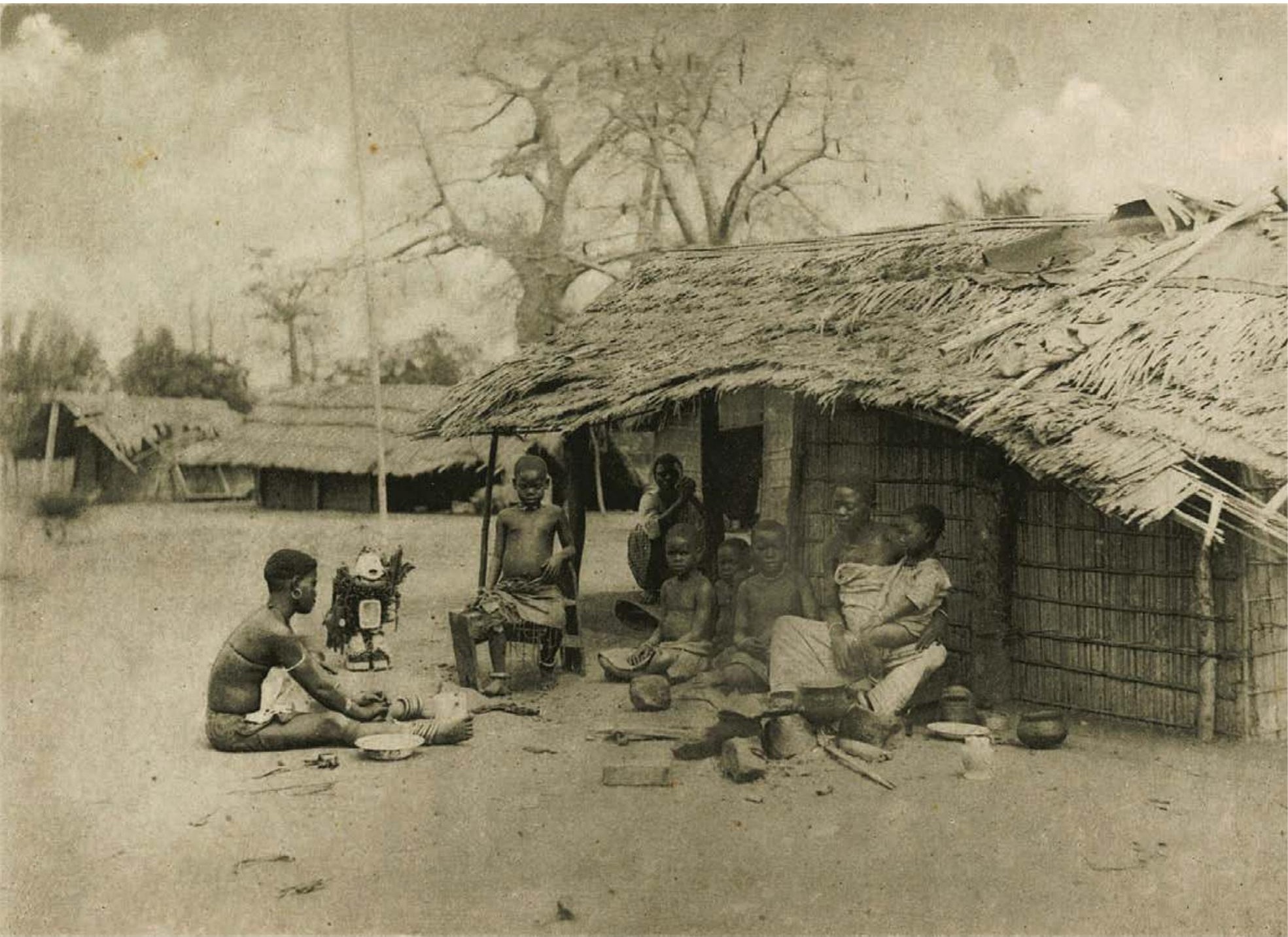

Fig. 10 Séance de divination. En retrait, à gauche, un nkisi nkondi, in J. A. da Cunha Moraes, Africa Occidental, Lisbonne, David Corazzi, 1885. 


\section{CARL EINSTEIN ET LES PRIMITIVISMES}

- $\bullet$

20. Dans un des rares passages d'Afrikanische Plastik reprenant Negerplastik, Einstein livrait un développement répréhensible de cette conception et terminait par cette formulation, qui pourrait servir de résumé de Und Afrika sprach : «Les forces créatrices de la civilisation africaine sont presque complètement épuisées. Peu à peu, la colonisation a détruit l'ancienne tradition, et les apports étrangers se sont mêlés aux trésors héréditaires des idées originales. 》 (1922 [1921] : 3)

21. Einstein, 1915 : xi ; trad. Meffre, 1998 : 19; Frobenius, Und Afrika sprach, vol. I [1912-1913) : chap. I. Mais Aloïs Riegl aussi aurait pu être ici une source théorique, comme le rappelle astucieusement Joyce Cheng (2009: 88 n. ?).

22. « On donne souvent le nom de fétiche aux statues africaines; mais ce terme, dont on fait un emploi abusif, finit par perdre sa signification véritable et ne sert souvent qu'à cacher notre ignorance. 》 (Einstein, 1922 [1921]:6] mais ne semblait pas moins convaincu par l'idée d'un déclin sans fin ${ }^{20}$. Notons au passage que la plupart des illustrations choisies par Einstein témoignaient de la vitalité de l'art africain contemporain.

En dépit des problèmes qu'il pose, Und Afrika sprach rendit disponible une immense quantité de données qui remirent en cause des schémas antérieurs sur les sociétés africaines. Le livre dévoilait une complexité historique qui rendait indéfendable la vision des Africains comme "peuple d'une préhistoire éternelle", selon les mots d'Einstein. Nul besoin de remonter en deçà de Frobenius pour retrouver l'idée d'Einstein selon laquelle il existait une histoire de l'art africain et une critique de l'affirmation illusoire selon laquelle «simple et originel [étaient] éventuellement identiques ${ }^{21} »$.

Cependant, la section qu'Einstein consacre dans Negerplastik à «la religion et l'art africain " ne dérive sans doute pas de Frobenius, ni d'ailleurs d'aucun autre travail savant sérieux de son temps. Einstein commence en affirmant que «l'art du Nègre est avant tout déterminé par la religion. Les œuvres sculptées sont vénérées comme elles le furent par tout peuple de l'Antiquité. L'exécutant [der Verfertiger] façonne son œuvre comme si elle était la divinité. » (Einstein, 1915 : xiii; trad. Meffre, 1998: 28) Il n'est pas difficile d'entendre là la jalousie, le désir, qu'éprouvait Einstein envers l'artiste africain (et il utilise bien le mot d'« artiste ", Künstler) qui crée un dieu et dont l'œuvre est «indépendante, transcendante et dégagée de tout lien" (ibid. : xiii; 29). L'artiste africain n'est pas mandaté pour imiter la nature, comme dans la tradition européenne : "Qui donc un dieu pourrait-il imiter, à qui pourrait-il se soumettre? " L'œuvre d'art africaine, elle, "ne signifie rien, elle n'est pas symbole; elle est le dieu » (ibid. : xv; 31).

Einstein n'emploie à aucun moment le mot «fétiche ${ }^{22}$ ». Mais ne nous y trompons pas. L'œuvre qui fait s'effondrer le signifiant et le signifié, cette chose que l'on prend pour un dieu, n'est rien d'autre que le «fétiche». En 1961, Jean Laude écrivit qu'il était inacceptable d'assimiler la sculpture africaine et le fétiche, mais il attribua l'erreur d'Einstein à la faiblesse de l'ethnographie de l'époque (1961: 88). Cette déclaration a servi d'alibi à d'innombrables défenseurs d'Einstein; mais elle renvoie une image fausse de la situation telle qu'elle se présentait en 1914. Des images sensationnalistes d'indigènes en adoration devant de grotesques fétiches continuaient de se répandre dans la presse populaire et dans les bandes dessinées, mais elles avaient pour ainsi dire disparu des pages de la littérature savante. Les formules d'Einstein citées plus haut évoquent Charles de Brosses qui, en 1760, écrivait que «[c]es fétiches divins ne sont autre chose que le premier objet matériel qu'il plaît à chaque nation ou à chaque particulier de choisir... Ce sont autant de dieux» (De Brosses, 1760 : 18, 19). Si l'on en croit l'Oxford English Dictionary, c'est de Brosses qui, le premier, affirma que le fétiche était « adoré en vertu de son caractère propre, non comme l'image, le symbole ou la résidence temporaire d'une divinité». C'est précisément sur cette distinction qu'Einstein met l'accent - la nature prétendument non symbolique, non référentielle du fétiche.

De Brosses fut discrédité dans les années 1870, quand les Européens se mirent à interroger des pratiquants des cultes et furent confrontés à la complexité des pratiques religieuses africaines. Dans son ouvrage célèbre, The Origin of Civilisation, John Lubbock trace une ligne d'évolution des peuples primitifs qui, partie des peuples sans religion, passe par le fétichisme «nègre» et s'achève avec l'émergence de la religion dans le totémisme (1871: 349-351). Il ne parvient pas à trancher la question de savoir si le «fétichisme » est un stade premier de reli- 
gion ou bien "d'anti-religion ", car «le Nègre croit que par l'entremise du fétiche il peut contraindre et contrôler la divinité " (ibid. : 164). Lubbock associe le fétiche aux images de sorcellerie de la tradition européenne, qui pouvaient être utilisées pour nuire à l'individu figuré (ibid. : 164, 165). Selon la conception de Lubbock, l'Africain, "par l'entremise de la sorcellerie, s'efforce de faire de la divinité son esclave" (ibid. : 349). Cette perspective est l'exact inverse de celle d'Einstein, qui imagine le sculpteur en train de fabriquer un objet-dieu, vouant un culte à cet objet qui, au final, le « consume».

En 1871, E.B. Tylor proposa une nouvelle définition du "fétichisme» qui fit date. Pour lui, le fétichisme était «la doctrine des esprits... attachés à certains objets matériels ou exerçant une influence par l'entremise de ces objets " (vol. II : 144). Un autre texte qui rencontra lui aussi un écho important, Notes analytiques, contesta l'existence d'une telle relation aux esprits. Comme dans bon nombre de publications sérieuses sur l'Afrique parues au début du xx siècle, ses auteurs se donnèrent pour objectif de renverser les images de la religion africaine qui avaient cours dans la presse de l'époque. Ils affirmèrent avec insistance et de la façon la plus explicite : " Les fétiches ne sont pas des idoles, ils ne sont pas des êtres ou des objets auxquels on rend un vrai culte. » (1906:149) «[C]'est une image, une effigie, un symbole investi d'une puissance temporaire. » (Ibid. : 151) «[J]amais [le Noir] ne se prosternera devant un fétiche... On ne l'adore pas comme une idole, comme un dieu. " (Ibid. : 160) Notes analytiques fut la publication définitive du musée du Congo et a par la suite servi de guide de référence pour les départements de recherche des musées de par le monde ainsi que pour des chercheurs tel Matvejs. Chez les auteurs cités, l'analyse était incomplète, les interprétations contradictoires, le ton condescendant, mais tous s'attaquaient à la réalité à un niveau bien plus complexe que ne le faisait Einstein.

Je voudrais insister sur le fait qu'Einstein avait choisi un modèle anachronique de manière délibérée et consciente. Il n'est donc pas surprenant qu'il l'ait abandonné totalement dans ses textes ultérieurs ${ }^{23}$. Replacé dans le cadre d'une réflexion sur "la pure sculpture ", le concept du fétiche existant seulement pour lui-même fournit un modèle important aux théories de l'objet d'art autonome défendues par les critiques européens à partir des années 1870.

\section{Dans le noir?}

On ne dispose à ce jour d'aucune information sur les sources de Negerplastik ${ }^{24}$. On est dès lors contraint d'analyser avec précision le texte lui-même. Une phrase étrange d'Einstein, répétée deux fois, fournit un indice révélateur : «Le fidèle adore souvent les objets dans l'obscurité.» (1915: xiv; trad. Meffre 1998: 29) À partir des années 1870 , les récits de voyage et les premières monographies ethnographiques sur l'Afrique furent publiés avec un nombre toujours croissant de gravures, puis de photographies. L'affirmation d'Einstein est dès lors problématique, car une simple consultation rapide de ces ouvrages révèle un certain nombre d'illustrations montrant des cérémonies où les masques sont exposés en plein jour, ou bien des expositions publiques d'œuvres d'art 25 (fig. 10).

C'est l'obscurité qui déclenche "la crainte du dieu» (Grauen vor dem Gott) évoquée par Einstein (ibid. xIII : 28). Selon Matthew Rampley, le «sublime est un trope fondamental des théories sur les cultures primitives et, en particulier, des théories de l'art primitif et préhistorique» (2005: 251). Entre 1876 et 1903, l'image
-

23. Dans Afrikanische Plastik, son modèle est par exemple «le culte des ancêtres », tel qu'il avait été développé par Bernhard Ankermann en 1918 [1922 [1921] : 13ff]. L'accent mis sur les «ancêtres » ouvre peut-être la porte à une analyse historique.

24. Joyce Cheng a identifié le texte d'Hedwig Fechheimer de 1914, Die Plastik der Ägypter, comme un livre précurseur (2009: 90). Je ne suis pas convaincue par d'autres suggestions tirées de la bibliographie d'Afrikanische Plastik, pour des raisons qui sont explicitées ailleurs dans cet article.

25. Joyce Cheng interroge le rôle de l'obscurité dans la théorie formaliste d'Einstein et va jusqu'à dire que celui-ci fut capable de «déduire » de la structure formelle de la sculpture africaine «la hiérarchie de valeur qui fait primer la présence sur l'apparence ». C'est là un jeu dangereux. Cheng dresse des parallèles avec les conceptions de Susan Vogel sur la stricte réglementation de la visibilité de l'art chez les Baoulé [1997). Cependant, même chez les Baoulé, le propriétaire rituel des sculptures auxquelles l'accès est restreint peut les prendre dans ses mains, les caresser et les regarder de près. La restriction de la distance et du temps d'accès aux œuvres n'est pas un équivalent du culte dans le noir évoqué par Einstein. 


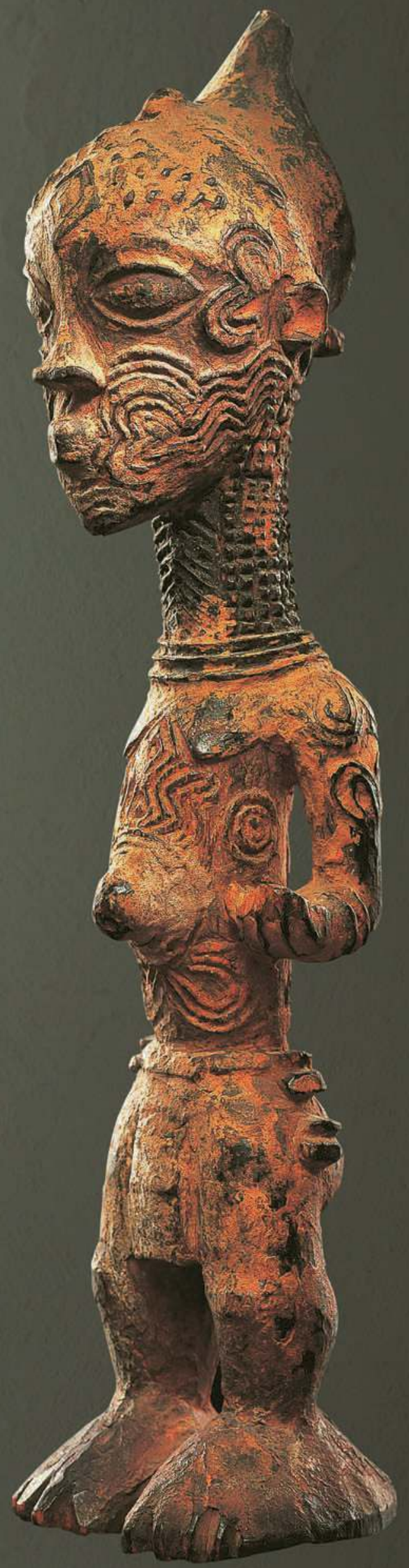

des hommes de la préhistoire changea profondément. Ils n'étaient plus de nobles sauvages, cherchant leur nourriture dans des prairies et des forêts fertiles, mais des créatures pitoyables luttant pour leur survie dans un monde plein de dangers (Groenen, 1994:328, 329). La radicalité de ce changement est patente dans la série de romans de J.-H. Rosny sur la vie dans la préhistoire, dont le plus célèbre, $L a$ Guerre du feu, décrit en détail les périls que doit affronter une famille dont le feu vient de s'éteindre et qui se retrouve plongée dans «la nuit épouvantable» (1911).

L'obscurité fut en particulier au cœur des débats qui entourèrent la découverte des peintures paléolithiques, à commencer par celles d'Altamira en 1880. Les archéologues français discutèrent l'authenticité de ces fresques jusqu'en 1902, date à laquelle le respectable Cartailhac se rétracta de façon dramatique en publiant son "Mea Culpa». Il ne pouvait concevoir qu'avec beaucoup de difficulté que quelqu'un ait pu réaliser des œuvres d'une telle qualité "dans ces antres obscurs, à la lumière vacillante de ces lampes fumeuses » (1902: 349).

Une fois l'authenticité des peintures murales admise par la communauté scientifique, l'obscurité resta le seul critère vraiment déterminant qui orientait toutes les interprétations. En 1903, Salomon Reinach écrivait que la localisation des peintures «dans la partie la plus obscure de la caverne » rendait leur "caractère religieux et mystique [...] incontestable» (1903: 263). Comme Lubbock, que l'on a évoqué plus haut, Reinach reprenait des conceptions développées à propos de la sorcellerie européenne et interprétait dès lors l'image (ou l'« effigie») comme un moyen d'influencer l'objet de la représentation ou d'exercer son pouvoir sur lui (ibid. : 260). Il s'autorisa prudemment des analogies avec les découvertes ethnographiques de son époque sur les Arunta d'Australie (qui réalisent, pour la multiplication du gibier, des peintures censées rester cachées aux noninitiés) : «Si les troglodytes pensaient comme les Arunta, les cérémonies qu'ils accomplissaient devant ces effigies devaient tendre à assurer la multiplication des éléphants, des taureaux sauvages, des chevaux, des cervidés, qui leur servaient ordinairement de nourriture " (ibid. : 263). Avec la profusion d'illustrations en couleurs dans les publications des années 1910, les artistes du paléolithique étaient très en vogue. L'interprétation de Reinach selon laquelle les peintures étaient des instruments de "magie cynégétique" (selon l'expression plus tardive) fut dominante pendant la première moitié $d u x^{e}$ siècle. Tout en s'opposant au modèle d'un artiste cherchant à contrôler la représentation, Einstein était porté à croire que l'obscurité était une des clés de l'interprétation, une thèse que, concernant l'Afrique, les savants de l'époque ne pouvaient approuver. 
Negerplastik se termine sur une discussion portant sur les tatouages et les masques; on n'a pas encore reconnu toute l'originalité de ce passage. Quand le capitaine James Cook publia son récit de voyage à Tahiti en 1769, il inaugura une véritable obsession de l'Europe pour les tatouages, qui dura jusqu'au début du xx siècle. Plusieurs voyageurs à la sensibilité scientifique décrivirent avec soin les individus tatoués (hommes, femmes, guerriers, etc.) et répertorièrent les endroits du corps où figuraient les dessins. À partir de cette abondante littérature, Lubbock conclut : "L'ornementation de la peau est un phénomène quasi universel parmi les races humaines inférieures. " (1871: 43) Les Maoris possédaient selon lui les tatouages "les plus beaux de tous " (ibid. : 47) et ses illustrations furent reprises, entre autres, par Aloïs Riegl. Le tatouage revêtait une très grande importance parce que, comme l'indiquait Lubbock, on pensait qu'il apportait une clé décisive pour l'histoire de l'ornement et, par extension, à la question de l'origine de l'art ${ }^{26}$. Riegl estimait que "l'impulsion de décorer [...] est l'un des instincts les plus élémentaires de l'homme » et il faisait référence aux Polynésiens pour démontrer que le tatouage avait été inventé avant le vêtement (1992 : 31). Dans l'une de ses plus célèbres déclarations, l'architecte moderniste Adolf Loos affirma que l'on pouvait mesurer le degré d'évolution humaine à la volonté de renoncer à l'ornement : «Le Papou tatoue sa peau, son embarcation, ses rames, en somme tout ce sur quoi il peut mettre la main. Ce n'est pas un criminel. L'homme moderne qui se fait tatouer est un criminel ou bien un dégénéré. Dans certaines prisons, quatre-vingts pour cent des prisonniers portent des tatouages. [...] Si un individu tatoué meurt en liberté, cela veut dire qu'il est mort quelques années avant de commettre un meurtre ${ }^{27}$." (1964: 19) Loos était d'accord pour dire que l'art naissait de "l'impulsion de décorer " le corps pour des motifs «érotiques ». Le succès des conférences données par Loos témoignèrent de la pertinence du sujet à l'époque d'Einstein.

Les Africains n'occupaient pas une place centrale dans la littérature théorique sur le tatouage, même si la scarification était à l'époque rangée dans la même catégorie générale. De nombreuses sculptures présentées dans Negerplastik étaient porteuses de scarifications, mais Einstein se contentait d'évoquer le corps humain et ce que ses transformations révélaient quant à la psychologie de l'artiste. Dans son texte, on trouve quelques traces de la littérature sur le tatouage, par exemple des références à sa "puissance érotique" (Kraft der Erotik) ou à la question de savoir si le motif "renforce» "la forme esquissée par la nature».

Cependant, dans Negerplastik, Einstein livre dans un texte au style très recherché, à la fois "laconique et expressionniste ${ }^{28}$ ", une interprétation sur le tatouage qui, à l'époque, était totalement inédite. Il est frappé par le fait suivant : "Quelle prise de conscience cela représente de concevoir son propre corps comme une œuvre inachevée que l'on transforme sans plus attendre! » (1915 : xxv; trad. Meffre, 1998 : 44) Encore une fois, il ne peut attribuer qu'à une «religion despotique » cette volonté de puissance qui ne recule devant rien pour "transformer par le tatouage le corps individuel en un corps collectif "[den individuellen Leib durch Tätowierung zu einem allgemeinen machen]. Selon lui, la capacité de se percevoir comme un objet, comme un médium, autorise un «don prodigieux de création objective » (ibid. ; 44-45).

Dans ce passage, Einstein essaie de lier ensemble la notion anachronique du fétiche, insistant sur la surévaluation des choses et son caractère arbitraire, et la littérature sur le totémisme qui voyait alors le jour. Selon lui, on peut com-
PAGE CI-CONTRE

Fig. 11 Statuette scarifiée lulua (c) musée Barbier-Mueller, photo studio Ferrazzini Bouchet.

\section{-}

26. Par exemple Hirn, 1900 et Grosse, 1902. Sur le débat autour de l'ornement, voir Rampley, 2005 : 255, 256.

27. Loos, 1964 : 19. Loos publia d'abord son article en français en 1913 («0rnement et Crime 》). Mais il avait déjà donné des conférences publiques sur le sujet à Berlin à partir de 1909 et avait développé la «substance de ses hypothèses » dès le tournant du siècle. Voir Long, 2009, pour la chronologie précise des conférences et de l'article mettant en forme ces hypothèses.

28. Je remercie Eberhard Fischer pour la perspicacité de ses remarques sur la dernière section de Negerplastik. 
29. Lévy-Bruhl (1910: 105). Avant ce passage, Lévy-Bruhl parle de blizzards, de brises, de vents, de fleuves et de tigres (102-105). Faut-il voir là l'origine des transformations en «un chat, un fleuve et le temps qu'il fait », énumérées par Einstein (137)? prendre que quelqu'un qui "se sent chat, rivière, temps qu'il fait, se transforme; il est ce qu'il ressent et en assume les conséquences " (ibid.; 45). Ce n'est pas un hasard si Einstein emploie sept fois des mots qui évoquent le "changement " et la "transformation" dans le court paragraphe qu'il consacre aux masques (verändern; verwandeln; Verwandlung). Baldwin Spencer et F.J. Gillen, les auteurs de Natives Tribes of Central Australia, utilisent eux aussi trente-cinq fois le verbe "transformer " et ses dérivés : animaux, oiseaux et larves peuvent tous prendre la forme d'humains, et inversement.

Pendant vingt ans, les publications de Spencer et Gillen exercèrent une influence considérable sur la vie intellectuelle européenne, car elles offraient des témoignages oculaires de la vie et de la culture des Arrernte (Arunta) d'Australie, présentés comme les survivants de l'âge de pierre. Les deux auteurs fournirent le matériau à partir duquel James George Frazer, Émile Durkheim, Lucien Lévy-Bruhl, Salomon Reinach, Henri Breuil et bien d'autres élaborèrent par la suite leurs théories fameuses sur le totémisme et la psychologie de la «mentalité primitive $"$.

En 1902, Durkheim et Marcel Mauss affirmèrent que les histoires de «métamorphoses» se retrouvaient dans le monde entier : "Or ce qu'elles supposent, c'est la croyance en la transformation possible des choses les plus hétérogènes les unes dans les autres.» (1902: 3, 4) À la lumière des travaux de Spencer et Gillen et d'autres ethnologues, ils firent l'hypothèse suivante à propos des peuples les moins évolués : «Ici, l'individu lui-même perd sa personnalité. Entre lui et son âme extérieure, entre lui et son totem, l'indistinction est complète. Sa personnalité et celle de son fellow-animal ne font qu'un. [...] C'est de bonne foi que le Bororo s'imagine être en personne un arara. " (Ibid. : 4) Dans ce modèle, dont l'influence fut très grande, la «transformation" repose sur l'extinction de la personnalité humaine.

En 1910, Lévy-Bruhl nuança ce modèle et tira des conclusions différentes en s'appuyant en grande partie sur les mêmes sources. Selon lui, il s'agissait plutôt d'une "fusion" ou d'une "participation mystique " quand un homme revêtait la peau d'un animal: "Ils ne s'occupent pas de savoir si l'homme cesse d'être homme pour devenir tigre, et ensuite d'être tigre pour redevenir homme. Ce qui les intéresse, c'est la vertu mystique qui rend ces individus participables [...] à la fois du tigre et de l'homme ${ }^{29}$."

Dans les années 1930, Einstein accorda une place très importante aux théories de Lévy-Bruhl mais, dans Negerplastik, sa préférence allait à la perspective de Durkheim et Mauss sur la psychologie de la transformation. Au lieu de parler de fusion, selon la conception de Lévy-Bruhl, il insistait sur le fait que toute individualité est "réduite à néant» (1915 : xxvi; trad. Meffre, $1998: 45)$. Le masque est sans expression parce qu'il est libéré de " toute expérience individuelle " (ibid.). Le porteur du masque devient le dieu. Pour Einstein, les cérémonies avec masques présentent des enjeux plus grands que le tatouage, car c'est la danse qui mène à l'extase. La cérémonie compense l'effacement de l'individu, implicite dans le culte religieux : l'individu prie le dieu, il danse en extase pour le clan (ou la communauté) et «il se transforme lui-même au moyen du masque en cette tribu et en ce dieu» (ibid.). À travers l'accent mis sur «le dieu», on entend résonner le fétiche. Lévy-Bruhl a défini l'extase comme un des «états limites, où, la fusion devenant complète entre le sujet et l'objet, la représentation proprement dite disparaît» (1910: 426). Einstein parle quant à lui du masque comme une "extase fixée" (fixierte Ekstase), signifiant par là que le masque saisit sur le vif le passage fugace de l'extase. Certains masques à l'expression grotesque, en particulier, sont 
capables de communiquer l'expérience de la transformation (1915 : xxvi). Einstein va même jusqu'à se demander si le fait de revêtir le masque ne peut pas servir de «stimulus» à l'extase.

Il faut noter icil'accent mis par Einstein sur l'expérience du porteur du masque. Ses prédécesseurs s'intéressaient davantage au public assistant à ces cérémonies. Dans ses premiers travaux, Frobenius estimait ainsi que les masques étaient des représentations des morts, qui étaient animées au cours de la performance (Streck, 1995 : 256). Pour Frazer, dans Le Rameau d'or, les cérémonies faisant intervenir les masques étaient chargées de donner une représentation réaliste des dieux pour renforcer la croyance de l'assistance (1913, section VI : 374-375). Les spéculations d'Einstein sont dès lors une expression précoce de ce que l'historien des religions Henry Pernet (1992: 117) a identifié comme une rupture brutale dans les études sur les cérémonies à masques : l'irruption d'une fascination nouvelle pour la psychologie du porteur de masque.

Entre les années 1930 et 1960, plusieurs grands théoriciens appliquèrent les théories de la psychologie de la «mentalité primitive" au masque et à ses rites (Lévy-Bruhl, 1963 [1931], Eliade, 1964, Buraud, 1948 et Caillois, 1958). En 1964, Mircea Eliade résuma les positions de ces chercheurs en des termes qui rappellent étonnamment ceux d'Einstein: "Le porteur du masque, quelle que soit sa fonction, transcende le temps historique. Ou'il soit rituel, funéraire ou destiné à tout autre spectacle, le masque est un instrument d'extase. Celui qui le porte n'est plus lui-même, puisqu'il est projeté au-delà de son identité personnelle temporelle.» (1964, vol. IX : 524) La bibliographie qui accompagnait la célèbre notice que rédigea Eliade sur «Les origines mythologiques et rituelles» des "Masques" de l'Encyclopedia of World Art (Encyclopédie de l'art mondial) faisait référence à six travaux sur les masques d'Europe centrale, au livre de Roger Caillois sur Les Jeux et les hommes et à un seul texte sur l'Afrique (le premier livre de Frobenius, qui n'était pas issu d'un travail de terrain; ibid. : 568).

De fait, ce n'est que dans les années 1970 que l'hypothèse de la transformation fit son apparition dans des travaux issus de

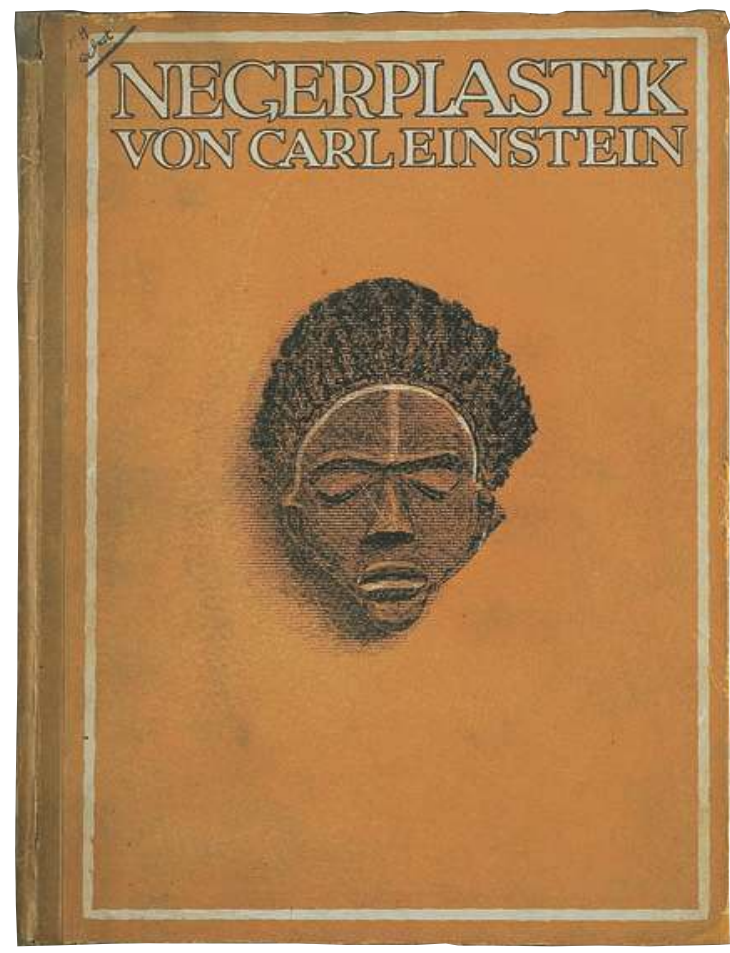

Fig. 12 Carl Einstein, couverture de l'édition originale de Negerplastik, Munich, Kurt Wolff Verlag, 1920. Photo Alberto Ricci. terrains réalisés en Afrique. L'une des figures majeures de ce revirement de situation fut Herbert Cole qui, en 1970, organisa une exposition intitulée African Arts of Transformation (Les Arts africains de la transformation). En 1985, il publia un volume collectif réunissant des textes d'étudiants sous le titre I am not Myself. La préface qu'il rédigea pour cette publication modeste est le texte le plus couramment cité sur les apparitions de masques dans les cérémonies africaines. Les chercheurs anglophones, écrit-il, laissent entendre que, "par l'entremise du masque ou du costume, un esprit est représenté. Ce n'est pas la conception des Africains. [...] L'individu masqué, le porteur du masque, qui est maintenant "possédé" ou pénétré par l'esprit, croit lui aussi à ce nouvel état altéré qui est le sien. Son caractère et son comportement personnels se trouvent modifiés et fusionnent avec ceux de l'esprit qu'il crée et qu'il devient. L'individualité humaine le quitte. Il n'est pas lui-même. " (Cole, 1985 : 20) Comme le fait remarquer Pernet, il existe de nombreuses versions fondées sur d'excellentes sources qui vont à l'encontre de cette théorie (1992 : 133-135). On s'étonne dès lors qu'elle soit présentée ici comme 


\section{CARL EINSTEIN ET LES PRIMITIVISMES}

$\bullet \bullet$

30. Communication personnelle, UCLA, printemps 2005. Cheng cite Cole pour donner raison à Einstein (2009: 100) ; pour ma part, je considère Cole comme un descendant intellectuel lointain d'Einstein.

31. Voir Strother, Z.S., « Dancing a Topic to Death : After 100 years of Research, What Do We Really Know about Masquerade in Africa? », communication au colloque Visualizing Africa, University of Michigan Museum of Art, 2002.

32. Je remercie Jonathan Fine d'avoir attiré mon attention sur l'importance des termes philosophiques dans Negerplastik.

33. Kiefer a fait une analyse superbe des sources de l'usage du mot «métamorphose » dans le travail d'Einstein [1987 : 159164). Pour lui, l'apparition du terme signale un renversement de la méthode formaliste ou ethnologique d'Einstein, tandis que, pour ma part, j'estime qu'elle prolonge son intérêt pour la «transformation 》. Sur la métamorphose, voir Lichtenstern 1998 et Zeidler 2010. une règle générale ("la conception des Africains»). Cole avait mené un important travail de terrain chez les Igbo du Nigeria, mais sa recherche ne portait pas principalement sur les masques. Dans des entretiens, il reconnut qu'il s'était appuyé sur Eliade (dont les écrits apparaissent en filigrane), mais il maintint sa position $^{30}$.

Dans une publication future, je souhaite retracer la généalogie des théories sur les cérémonies à masques en Afrique ${ }^{31}$. À l'exception peut-être d'Eliade, il semblerait que personne n'ait lu le texte d'Einstein. À la lecture de ce dernier, il apparaît clairement que l'hypothèse de la transformation n'avait pas été formulée de prime abord à propos de l'Afrique. Il s'agissait d'une transposition des théories de la "mentalité primitive" au cas des cérémonies à masques, notamment de la conception selon laquelle les hommes étaient transformés en totem soit par "fusion ", soit par aliénation complète de leur personnalité.

Il est important de souligner ici qu'Einstein ne s'appuyait pas sur l'ethnographie africaine mais sur un mélange de sources explorant ce qu'on appelait alors la «mentalité primitive ». Cette dimension est en effet révélatrice de son projet. Pour beaucoup de chercheurs, Negerplastik est une énigme : Einstein fonde sa méthode sur ce qu'il décrit comme une "analyse des formes" (Analyse der Formen; 1915 : viii), mais son étude est parcourue de longues exégèses sur la religion et la psychologie. Le problème vient peut-être de la confusion entre l'« analyse des formes " telle que la concevait Einstein et la description brute que l'on désigne aujourd'hui sous le terme d'« analyse formelle». Einstein cherchait à capter «les manières de voir et les lois de la vision" (Sehweisen und Gesetze der Anschauung; 1915: viii; trad. Meffre, $1998: 21$ ). Le mot allemand Anschauung possède un sens à la fois physique et philosophique ${ }^{32}$. À la fin de sa note méthodologique, Einstein reconnaît la nature "arbitraire» de la création artistique qui découle des «formes individuelles de vision" (die einzelnen Formen der Anschauung). Cette dernière est ainsi façonnée par la culture et par la psychologie. Dès lors qu'Einstein souhaitait saisir les «manières de voir» des Africains à partir de leurs sculptures, il lui incombait d'explorer le type de psychologie qui les avait produites.

Un dernier mot sur la transformation : «Voir ne signifie plus observer... »

Einstein poursuivit son travail sur la transformation tout au long de sa vie. Sa réflexion la plus poussée et la plus machiste sur le primitivisme figure curieusement dans sa monographie sur Georges Braque ${ }^{33}$. Dans ce texte, le terme "fétichiste» est péjoratif et sert à se moquer des esthètes européens qui vénèrent l'objet d'art et en ont secrètement peur ("à la manière des primitifs "; Einstein 1934 :13, 54). À partir de 1930, sous l'influence d'une critique jungienne de Sigmund Freud, Einstein affirma que l'inconscient ne devait pas être considéré comme une force négative : «Nous croyons par contre que c'est précisément dans l'inconscient que réside la chance du Nouveau; qu'il se reforme sans cesse et qu'on peut le considérer aussi comme un facteur progressif. " (Ibid. : 118) Pour Einstein, Braque est un visionnaire parce qu'il explore l'inconscient à travers le rêve ou l'hallucination et a le courage de mener ce travail seul, sans le soutien de la religion ou de la solidarité collective des "primitifs ». Bien qu'Einstein ne fasse pas référence à l'Afrique dans ce texte, il invoque la «rupture extatique " du porteur de masque et rappelle que l'animiste ou le totémiste est "dominé par le besoin de détruire sa personne [...], dominé, en d'autres mots, par le principe métamorphe » 
(ibid., 137-138). Braque vit désormais ce "drame», qui l'a libéré du besoin d'imiter la nature : "l'art devient un moyen magique, une force qui transforme le réel " (ibid., 139). Le Braque de la maturité a fait l'expérience de la «transformation de la vision » : "Voir ne signifie plus observer » (ibid., 140). Le désir qu'exprimait Negerplastik est maintenant plus clair. Einstein a ainsi passé sa vie à étudier les formes qu'il admirait en Afrique et ailleurs, afin d'arriver à maîtriser la psychologie dont elles étaient le produit.

\section{Columbia University, département d'histoire de l'art et d'archéologie zss1@columbia.edu}

Traduction de l'anglais par Camille Joseph

mots clés / keywords : primitivisme // primitivism - photographie de la sculpture // photography of sculpture - Carl Einstein // Carl Einstein • Negerplastik // Negerplastik • Vladimir Markov // Vladimir Markov • Iskusstvo Negrov // Iskusstvo Negrov.

\section{Résumé / Abstract}

Z.S. Strother, À la recherche de l'Afrique dans Negerplastik de Carl Einstein - La publication de Negerplastik de Carl Einstein constitua, pour le meilleur ou pour le pire, l'acte fondateur de l'histoire de l'art africain. Ce texte, qui proposait 119 photographies habilement présentées afin de permettre des comparaisons formelles, contribua à légitimer les catégories de «sculpture nègre» ou d'《art africain ». Einstein ne citait pourtant aucune étude contemporaine sur l'Afrique. II s'appuyait plutôt sur un mélange de différentes sources évoquant «la mentalité primitive». Dans l'un des passages les plus originaux de ce texte, il transférait le modèle du «totémisme » aux tatouages et aux masques. En faisant l'hypothèse que le porteur du masque, en passant de la danse à l'«extase », connaissait une expérience transformationnelle, Einstein préfigurait une évolution importante de la recherche sur les cérémonies avec masques. Son intérêt pour les textes traitant de la «mentalité archaïque » répondait à la tâche qu'il s'était fixée et qu'il concevait comme un projet aussi bien psychologique que formaliste : recouvrer «les modes de vision et les lois de perception » de l'art africain.
Z.S. Strother, Looking for Africa in Carl Einstein's Negerplastik For good or ill, the publication of Negerplastik constitutes a founding act of African art history. The presentation of 119 photographs in skillful formal comparisons made credible categories such as "Negro sculpture" or "African art." Nevertheless, Einstein did not cite any contemporary scholarship on Africa and instead drew upon a mixture of sources on the "primitive mind." In one of the most original passages, he transfers models for "totemism" onto tattoos and masks. In this respect, Einstein foreshadows an important shift in the literature on masquerade by hypothesizing a transformational experience for the performer who is led by dance into "ecstasy." Einstein's attraction to the literature on the "archaic mind" emerged from his mission to recover "ways of seeing and the laws of perception" for African art, a project he conceived as much psychological as it was formalistic. 